\title{
Decay Order of Eigenfunctions of Second-order Elliptic Operators in an Unbounded Domain, and its Applications
}

By

\author{
Jun UCHIYAMA*
}

\section{\$0. Introduction}

In this paper we shall study the asymptotic behavior as $|x| \rightarrow \infty$ of the not identically vanishing solution $u(x) \in H_{\text {loc }}^{2}\left(\mathbb{R}^{n}\right)$ of second-order elliptic equation in an unbounded domain

$$
\begin{gathered}
-\sum_{i, j=1}^{n}\left(\frac{\partial}{\partial x_{i}}+\sqrt{-1} b_{i}(x)\right) a_{i j}(x)\left(\frac{\partial}{\partial x_{j}}+\sqrt{-1} b_{j}(x)\right) u(x) \\
+\left(q_{1}(x)+q_{2}(x)\right) u(x)=0 \quad \text { for }|x|>R_{0} .
\end{gathered}
$$

Our main assumptions are the following: there exist some constants $\alpha<3$ and $\alpha-1<r_{0}<2$ such that

$$
\begin{aligned}
& \operatorname{curl} b(x)=o\left(r^{-(\alpha+1) / 2}\right), \\
& q_{2}(x)=o\left(r^{(1-3 \alpha) / 4}\right) \text { is a complex-valued function, } \\
& q_{1}(x) \text { is a real-valued function satisfying } \\
& \limsup _{r \rightarrow \infty}\left\{r^{\alpha}\left\langle\hat{x}, A(x) \nabla q_{1}(x)\right\rangle+\gamma_{0} r^{\alpha-1} q_{1}(x)\right\}<\infty .
\end{aligned}
$$

(Note that we do not exclude the case $\lim _{r \rightarrow \infty} q_{1}(x)=\infty$.) These conditions are generalization of the preceding paper Uchiyama [2], which treats the case $\alpha=1$ of this paper and includes more strict conditions than ones imposed in this paper. More detailed conditions are stated in $\S 1$. Then for any $\mu>0$ satisfying

$$
\limsup _{r \rightarrow \infty}\left\{r^{\alpha}\left\langle\hat{x}, A(x) \nabla q_{1}(x)\right\rangle+r_{0} r^{\alpha-1} q_{1}(x)\right\}<4^{-1} \mu^{2}(3-\alpha)^{2}\left(\gamma_{0}-\alpha+1\right),
$$

we have by Theorem 1.1 in $\S 1$

* Communicated by S. Matsuura, November 21, 1985.

Kyoto Institute of Technology, Matsugasaki, Sakyo-ku, Kyoto 606, Japan. 


$$
\lim _{R \rightarrow \infty} \exp \left(2 \mu R^{(3-\infty) / 2}\right) \int_{R<|x|<R+1}|u(x)|^{2} d x=\infty .
$$

Bardos and Merigot [1, Theorem 2.2 (1), p. 329, which seems to need some modifications of its statements] treats the case $a_{i j}(x)=\delta_{i j}, b_{i}(x)=0, q_{2}(x)=0$ and $0<\alpha \leq 1$. Also $[1, \S 3]$ treats the case $a_{i j}(x)=\delta_{i j}, b_{i}(x)=0, q_{2}(x)=0$ and $\lim _{r \rightarrow \infty} q_{1}(x)=\infty$ under the more strict conditions which include the assumptions on $\frac{\partial^{2}}{\partial r^{2}} q_{1}(x)$, and gives more detailed results than ours.

In $\S 1$ the assumptions and Theorems $1.1,1.2$ and 1.3 are explained. $\S 2$ and $\S 3$ cover the proof of Theorem 1.1. In $\S 4$ we prove Theorems 1.2 and 1.3. The tools of proofs are similar to Uchiyama [2]. In $\S 5$ we apply Theorem 1.3 to the not identically vanishing solution $u(x) \in H_{\text {loc }}^{2}\left(\mathbb{R}^{n}\right)$ of the equation $-\Delta u(x)+\left(q_{1}(x)+q_{2}(x)\right) u(x)=0$. There we consider several examples as follows:

$$
\begin{aligned}
& q_{1}(x)+q_{2}(x)=c r^{\theta}+o\left(r^{\rho}\right)-\left(\lambda_{1}+i \lambda_{2}\right) \quad(\text { Examples 5.1 5.3), } \\
& q_{1}(x)+q_{2}(x)=r^{\theta}\langle e, x\rangle+o\left(r^{\rho}\right)-\left(\lambda_{1}+i \lambda_{2}\right) \quad(\text { Example 5.4), } \\
& q_{1}(x)+q_{2}(x)=a r^{\sigma} \sin b r^{\tau}-\left(\lambda_{1}+i \lambda_{2}\right) \quad(\text { Example 5.5), } \\
& q_{1}(x)+q_{2}(x)=V(x)+o\left(r^{\rho}\right)-\left(\lambda_{1}+i \lambda_{2}\right) \quad(\text { Example 5.6) }
\end{aligned}
$$

where $c, \theta, \rho, \lambda_{1}, \lambda_{2}, a, b, \sigma, \tau(a b \neq 0, \tau>0)$ are real constants, $e \in \mathbb{R}^{n}(e \neq 0)$ is a constant vector, $i=\sqrt{-1}$, the function represented by $o\left(r^{\rho}\right)$ is a complexvalued one, and $V(x)$ is a real-valued function satisfying for some constant $r_{0} \leq 2$

$$
\limsup _{r \rightarrow \infty}\left[r^{\gamma_{0}+1} \partial_{r} V(x)+r_{0} r^{\gamma_{0}} V(x)\right] \leq 0 .
$$

Especially we have the following. If

$$
q_{1}(x)+q_{2}(x)=c r^{2}+o(r)-\lambda_{1} \quad\left(c>0, \lambda_{1}>0\right)
$$

(harmonic oscillator), we have for any $\varepsilon>0$

$$
\lim _{R \rightarrow \infty} \exp \left((1+\varepsilon) \sqrt{c} R^{2}\right) \int_{R<|x|<R+1}|u(x)|^{2} d x=\infty .
$$

If

$$
q_{1}(x)+q_{2}(x)=c r^{-1}+o\left(r^{-1 / 2}\right)-\lambda_{1} \quad\left(c \neq 0, \lambda_{1}<0\right)
$$

(hydrogen atom), or

$$
q_{1}(x)+q_{2}(x)=a r^{-1} \sin b r-\lambda_{1} \quad\left(a b \neq 0, \lambda_{1}<0\right)
$$


(adiabatic oscillator), or

$$
\begin{aligned}
q_{1}(x)+q_{2}(x)= & -\sum_{1 \leq i \leq N} Z_{i}\left|x^{(i)}\right|^{-1}+\sum_{1 \leq i<j \leq N} Z_{i j}\left|x^{(i)}-x^{(j)}\right|^{-1} \\
& +o\left(r^{-1 / 2}\right)-\lambda_{1} \quad\left(\lambda_{1}<0\right)
\end{aligned}
$$

where $Z_{i}$ and $Z_{i j}$ are real constants, $x^{(i)} \in \mathbb{R}^{3}$, and $x=\left(x^{(1)}, \cdots, x^{(N)}\right) \in \mathbb{R}^{3 N}$ (an atom with $N$ electrons), we have for any $\varepsilon>0$

If

$$
\lim _{R \rightarrow \infty} \exp \left(2(1+\varepsilon) \sqrt{-\lambda_{1}} R\right) \int_{R<|x|<R+1}|u(x)|^{2} d x=\infty .
$$

$$
q_{1}(x)+q_{2}(x)=\langle e, x\rangle+o\left(r^{1 / 4}\right)-\lambda_{1} \quad\left(-\infty<\lambda_{1}<\infty\right)
$$

(constant electric field), we have for any $\varepsilon>0$

$$
\lim _{R \rightarrow \infty} \exp \left(\frac{4}{3}(1+\varepsilon) \sqrt{|e|} R^{3 / 2}\right) \int_{R<|x|<R+1}|u(x)|^{2} d x=\infty .
$$

\section{§1. Notations amd $\mathbb{M}$ Min $\mathbb{R}$ esullts}

At first, we shall list the notations which will be freely used in the sequel, and are the same as given in Uchiyama [2].

\section{Notations:}

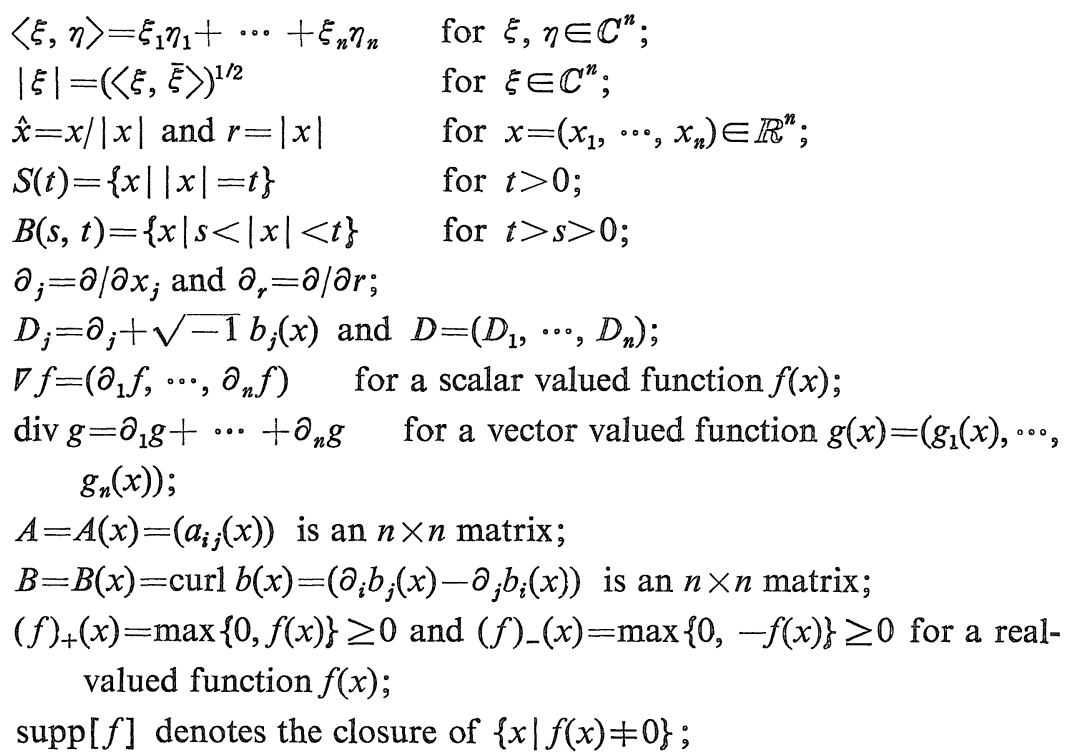
$C^{j}(\Omega)$ denotes the class of $j$-times continuously differentiable functions: $C_{0}^{\infty}(\Omega)=\left\{f(x) \mid\right.$ for any $j=0,1,2, \cdots, f \in C^{j}(\Omega)$ and $\operatorname{supp}[f]$ is a 
compact set in $\Omega$;

$L^{p}(\Omega)=\left\{\left.f(x)\left|\int_{\Omega}\right| f(x)\right|^{p} d x<\infty\right\} \quad$ for $p \geq 1 ;$ $L_{\mathrm{loc}}^{p}(\Omega)=\left\{\left.f(x)\right|^{\Omega}\right.$ for any compact set $\left.K \subset \Omega, \int_{K}|f(x)|^{p} d x<\infty\right\}$ for
$\quad p \geq 1 ;$

$H^{m}(\Omega)$ denotes the class of $L^{2}$-functions in $\Omega$ such that all distribution derivatives up to $m$ belong to $L^{2}(\Omega)$;

$H_{\mathrm{loc}}^{m}(\Omega)=\left\{f(x) \mid\right.$ for any compact set $\left.K \subset \Omega, f \in H^{m}(K)\right\}$;

Next we shall state the conditions required in the Theorems.

\section{Assumptions:}

(A1) each $a_{i j}(x) \in C^{2}(\Omega)$ is a real-valued function;

(A2) $a_{i j}(x)=a_{j i}(x)$;

(A3) $a_{i j}(x) \rightarrow \delta_{i j}$ as $|x| \rightarrow \infty$;

(A4) there exists some constant $C_{1} \geq 1$ such that for any $x \in \Omega$ and any $\boldsymbol{\xi} \in \boldsymbol{C}^{n}$ we have

$$
C_{1}^{-1}|\xi|^{2} \leq\langle A(x) \xi, \bar{\xi}\rangle \leq C_{1}|\xi|^{2} ;
$$

(A5) $\partial_{l} a_{i j}(x)=o\left(r^{-1}\right)$ as $|x| \rightarrow \infty$;

(B ) each $b_{i}(x) \in C^{1}(\Omega)$ is a real-valued function;

(C1) $q_{1}(x)$ is a real-valued function;

(C2) for any $w \in H_{\mathrm{loc}}^{1}(\Omega)$ we have $q_{1}|w|^{2} \in L_{\mathrm{loc}}^{1}(\Omega)$;

(C3) for any $w \in H_{\text {loc }}^{1}(\Omega)$ we have $\left|\nabla q_{1}\right||w|^{2} \in L_{\text {loc }}^{1}(\Omega)$;

(D1) $q_{2}(x)$ is a complex-valued function;

(D2) for any $w \in H_{1 \mathrm{oc}}^{1}(\Omega)$ we have $q_{2}|w|^{2} \in L_{\mathrm{loc}}^{1}(\Omega)$;

( E ) there exists some constant $R_{0}>0$ such that $\Omega \supset\left\{x|| x \mid>R_{0}\right\}$;

( F ) there exist some constants $\alpha, \delta$ satisfying $\alpha<3,0<\delta<2^{-1}(3-\alpha)$ and some real-valued function $r(x) \in C^{1}(\Omega)$ such that

(F1) $\alpha-1+\delta<\gamma(x)<2-\delta \quad$ for $|x|>R_{0}$;

(F2)

$$
\begin{aligned}
M^{(\delta)} \equiv\left\{\mu>0 \mid \limsup _{r \rightarrow \infty}\left[r^{\alpha}\left\langle\hat{x}, A(x) \nabla q_{1}(x)\right\rangle+r^{\alpha-1} \gamma(x) q_{1}(x)\right.\right. \\
+(2 \mu(3-\alpha))^{-1} r^{\left(3^{\alpha-1}\right) / 2}\left|q_{2}(x)\right|^{2} \\
\quad+(2-\delta-\gamma(x))^{-1} r^{\alpha-1}|B(x) A(x) x|^{2} \\
\left.\left.\quad-4^{-1} \mu^{2}(3-\alpha)^{2}(r(x)-\alpha+1)\right]<0\right\} \\
\neq \phi,
\end{aligned}
$$

where $\phi$ denotes the empty set;

(F3) $\partial_{r} r(x)=o\left(r^{(5-3 \omega) / 4}\right)$ as $|x| \rightarrow \infty$;

(F4) $\left(\nabla-\hat{x} \partial_{r}\right) r(x)=o\left(r^{(1-\infty) / 2}\right)$ as $|x| \rightarrow \infty$; 
(F5) $\partial_{r} \partial_{i} a_{i j}(x)=o\left(r^{(1-3 \alpha) / 4}\right)$ as $|x| \rightarrow \infty$;

(F6) $\left(\nabla-\hat{x} \partial_{r}\right) \partial_{i} a_{i j}(x)=o\left(r^{-(1+\alpha) / 2}\right)$ as $|x| \rightarrow \infty$.

Remark 1.1. The above assumptions applied to the case $\alpha=1$ are weaker than ones given in Uchiyama [2].

So as to state the results we shall require the following:

Definition 1.1. For a constant $\eta$ satisfying $0 \leq \eta \leq \delta$ where $\delta$ is given in (F), let

$$
\begin{aligned}
& M^{(\eta)}=\left\{\mu>0 \mid \limsup _{r \rightarrow \infty}\left[r^{\alpha}\left\langle\hat{x}, A(x) \nabla q_{1}(x)\right\rangle+r^{\alpha-1} \gamma(x) q_{1}(x)\right.\right. \\
&+(2 \mu(3-\alpha))^{-1} r^{(3 \alpha-1) / 2}\left|q_{2}(x)\right|^{2} \\
&+(2-\eta-r(x))^{-1} r^{\alpha-1}|B(x) A(x) x|^{2} \\
&\left.\left.-4^{-1} \mu^{2}(3-\alpha)^{2}(r(x)-\alpha+1)\right]<0\right\} \\
& \mu^{(\eta)}=\inf M^{(\eta)}(\geq 0) \\
& \mu^{*}=\lim _{\eta \downarrow} \mu^{(\eta)}(\geq 0) .
\end{aligned}
$$

Remark 1.2. Well-definedness of Definition 1.1 will be shown in Lemma 2.1.

Remark 1.3. The above definition is independent of (C3), and depends essentially on (F1) and (F2). So Theorems 1.1 and 1.2 use the above definition. On the other hand, since we weaken the condition $(F)$ in Theorem 1.3, we use the another definition of $\mu^{*}$, which can be considered as the one obtained in the case that $a_{i j}(x)=\delta_{i j}$ and $b_{i}(x)=0$ under the condition $(\mathrm{F})$.

Now we have the

Theorem $\mathbb{1}_{0} \mathbb{1}$. Let $u(x)$ satisfy

$$
\left(^{*}\right):\left\{\begin{array}{l}
-\langle D, A D u\rangle+\left(q_{1}+q_{2}\right) u=0 \text { in } \Omega, \\
u \in H_{\mathrm{loc}}^{2}(\Omega), \\
\operatorname{supp}[u] \text { is not a compact set in } \bar{\Omega}(\text { closure of } \Omega) .
\end{array}\right.
$$

Let conditions (A), (B), (C), (D), (E) and (F) hold. Then

(1) for any $\mu>\mu^{*}$ we have

$$
\lim _{R \rightarrow \infty} \exp \left(2 \mu R^{(3-a) / 2}\right) \int_{S(R)}\left[|\langle A D u, \hat{x}\rangle|^{2}+\left\{1+\left(q_{1}\right)_{-}\right\}|u|^{2}\right] d S=\infty .
$$

(2) Moreover we assume

$(\mathrm{G})$ there exist some constants $a, b, \nu^{*}, C_{2}$ satisfying $0<a<1,0<b$, $\nu^{*} \geq 0, C_{2} \geq 0$ such that for any $w \in H^{1}(\Omega)$ having a compact 
support in $\&$ we have

$$
\begin{aligned}
& \int_{\Omega}\left(q_{1}+\operatorname{Re}\left[q_{2}\right]\right)_{-}|w|^{2} d x \leq a \int_{\Omega}|\nabla w|^{2} d x+C_{2} \int_{\Omega} \exp \left(\nu^{*} r^{(3-\alpha) / 2}\right)|w|^{2} d x, \\
& \int_{\Omega}\left(q_{1}\right)_{-}|w|^{2} d x \leq b \int_{\Omega}|\nabla w|^{2} d x+C_{2} \int_{\Omega} \exp \left(\nu^{*} r^{(3-\alpha) / 2}\right)|w|^{2} d x,
\end{aligned}
$$

where $\alpha<3$ is given in $(\mathbb{F})$. Then we have for any $\mu>\mu^{*}+2^{-1} \nu^{*}$

$$
\lim _{R \rightarrow \infty} \exp \left(2 \mu R^{(3-\alpha) / 2}\right) \int_{B(R, R+1)}|u|^{2} d x=\infty .
$$

Now we shall consider the more special case under the weaker conditions.

Theorem 1.2。 Let $u(x)$ satisfy

$$
(* *):\left\{\begin{array}{l}
-\langle D, D u\rangle+\left(q_{1}+q_{2}\right) u=0 \text { in } \Omega, \\
u \in H_{10 c}^{2}(\Omega), \\
\operatorname{supp}[u] \text { is not a compact set in } \bar{\Omega} .
\end{array}\right.
$$

We assume conditions (B), (C), (D), (E) and (F) with $a_{i j}\left(x^{\prime}=\delta_{i j}\right.$ except for (C3). Instead of $(\mathrm{C} 3)$ we assume

(C3)' for any $w \in H_{1 \mathrm{oc}}^{1}(\Omega)$ we have $\left(\partial_{r} q_{1}\right)|w|^{2} \in L_{\mathrm{loc}}^{1}(\Omega)$. Then all the assertions (1) and (2) in Theorem 1.1 with $a_{i j}(x)=\delta_{i j}$ also hold.

Lastly we shall consider the most special case under the weakest conditions.

Theorem 1.3。 Let $u(x)$ satisfy

$$
(* * *):\left\{\begin{array}{l}
-\Delta u+\left(q_{1}+q_{2}\right) u=0 \text { in } \Omega, \\
u \in H_{\mathrm{loc}}^{2}(\Omega), \\
\operatorname{supp}[u] \text { is not a compact set in } \bar{\Omega},
\end{array}\right.
$$

where $\Delta$ is a Laplacian in $\mathbb{R}^{n}$. Let (C1), (C2), (C3)', (D1), (D2) and (E) hold. Instead of $(\mathbb{F})$, we assume

$(\mathrm{F})^{\prime}$ there exist some constant $\alpha, \delta$ satisfying $\alpha<3,0<\delta<3-\alpha$ and some real-valued function $r(x) \in C^{1}(\Omega)$ such that

$(\mathrm{F} 1)^{\prime} \alpha-1+\delta<\gamma(x) \leq 2$ for $|x|>R_{0}$;

(F2)'

$$
\begin{aligned}
M \equiv & \left\{\mu>0 \mid \lim _{r \rightarrow \infty} \sup \left[r^{\alpha} \partial_{r} q_{1}(x)+r^{\alpha-1} \gamma(x) q_{1}(x)\right.\right. \\
& \left.\left.+(2 \mu(3-\alpha))^{-1} r^{(3 \alpha-1) / 2}\left|q_{2}(x)\right|^{2}-4^{-1} \mu^{2}(3-\alpha)^{2}(r(x)-\alpha+1)\right]<0\right\} \\
\neq &
\end{aligned}
$$


(F3) $\partial_{r} r(x)=o\left(r^{\left(5-3^{\alpha}\right) / 4}\right)$ as $|x| \rightarrow \infty$;

$(\mathbb{F} 4)^{\prime}$ there exists some nonnegative function $p(x)$ satisfying

$$
\begin{aligned}
& p(x)=o\left(r^{(1-\alpha) / 2}\right) \text { as }|x| \rightarrow \infty, \\
& \left|\left(\nabla-\hat{x} \partial_{r}\right) r(x)\right| \leq(2-r(x))^{1 / 2} p(x) \text { for }|x|>R_{0} .
\end{aligned}
$$

Let $\mu^{*}=\inf M(\geq 0)$. Then all the assertions (1) and (2) of Theorem 1.1 with $a_{i j}(x)=\delta_{i j}$ and $b_{i}(x)=0$ also hold.

Remark 1.4. Let $\Omega=\left\{x|| x \mid>R_{0}\right\}$ and

$$
q_{1}(x)=\left|x^{(i)}\right|^{\sigma}
$$

where $\sigma$ is a real constant, $x^{(i)} \in \mathbb{R}^{3}$ and $x=\left(x^{(1)}, \cdots, x^{(N)}\right) \in \mathbb{R}^{3 N}(N \geq 2)$. Then we have for $x \in \Omega$

$$
\begin{aligned}
& \left|\nabla q_{1}(x)\right|=|\sigma|\left|x^{(i)}\right|^{\sigma-1}, \\
& \left|\partial_{\gamma} q_{1}(x)\right|=|\sigma|\left|x^{(i)}\right|^{\sigma} r^{-1} \leq R_{0}^{-1}|\sigma|\left|x^{(i)}\right|^{\sigma} .
\end{aligned}
$$

So if $\sigma \geq-1, q_{1}(x)$ satisfies (C3). And if $\sigma \geq-2, q_{1}(x)$ satisfies (C3)'.

\section{§2. Proof of Theorem $1.1(1)$}

In this section all the conditions $(A) \sim(\mathbb{F})$ are assumed. (Also see $\mathbb{R e}$ mark 1.3.) At first we study on $\mu^{(\eta)}$, which is given in Definition 1.1.

Lemma 2.1. We have the followings.

(1) For any $0 \leq \eta \leq \delta, M^{(\eta)} \neq \phi$ and $\left(\mu^{(\eta)}, \infty\right) \subset M^{(\eta)}$.

(2) For any $0 \leq \eta_{2} \leq \eta_{1} \leq \delta$, we have $0 \leq \mu^{\left(\eta_{2}\right)} \leq \mu^{\left(\eta_{1}\right)}$. Then $\mu^{*}=\lim _{\eta_{\ddagger} 0} \mu^{(\eta)}$ exists.

(3) If $|B(x) A(x) x|=O\left(r^{(1-\infty) / 2}\right)$ as $|x| \rightarrow \infty$, we have $\mu^{*}=\mu^{(0)}$.

Proof. Let $0 \leq \eta \leq \delta$. It is obvious that $\phi \neq M^{(\delta)} \subset M^{(\eta)}$. We can easily see $\left(\mu^{(\eta)}, \infty\right) \subset M^{(\eta)}$, if $\mu^{(\eta)} \in M^{(\eta)}$. Now we assume inf $M^{(\eta)}=\mu^{(\eta)} \notin M^{(\eta)}$. For any $\mu \in\left(\mu^{(\eta)}, \infty\right)$ there exists $\mu^{\prime}$ satisfying $0 \leq \mu^{(\eta)}<\mu^{\prime}<\mu$ and $\mu^{\prime} \in M^{(\eta)}$. Noting $(2 \mu(3-\alpha))^{-1}<\left(2 \mu^{\prime}(3-\alpha)\right)^{-1}$ and $-\mu^{2}<-\mu^{\prime 2}$, we have $\mu \in M^{(\eta)}$, which means $\left(\mu^{(\eta)}, \infty\right) \subset M^{(\eta)}$. The assertion (1) is thus proved. The assertion (2) is obvious. Lastly we shall show (3). By (2) we have $\mu^{*} \geq \mu^{(0)}$. For any $\varepsilon>0, \mu^{(0)}+\varepsilon \in M^{(0)}$. Since $|B A x|^{2} r^{\alpha-1}=O(1)$ as $r \rightarrow \infty$, there exists some $0<\eta_{\varepsilon}<\delta$ such that $\mu^{(0)}+\varepsilon \in M^{\left(\eta_{\varepsilon}\right)}$, which means $\mu^{\left(\eta_{\varepsilon}\right)}<\mu^{(0)}+\varepsilon$. Then we have $\mu^{*} \leq \mu^{(0)}$.

In the same way as Uchiyama [2], we give the following definition.

Defimition 2.1. Let $u(x)$ satisfy $\left(^{*}\right)$ given in Theorem 1.1. For real- 
valued functions $\rho=\rho(r) \in C^{2}\left(R_{0}, \infty\right), f=f(r) \in C^{1}\left(R_{0}, \infty\right)$ and $g=g(x) \in C^{1}(\Omega)$, let

$$
\begin{aligned}
& v(x)=\exp (\rho(r)) u(x), \\
& k_{1}(x)=-\left\{\rho^{\prime}(r)\right\}^{2}\langle A(x) \hat{x}, \hat{x}\rangle, \\
& k_{2}(x)=\rho^{\prime \prime}(r)\langle A(x) \hat{x}, \hat{x}\rangle+\rho^{\prime}(r) \operatorname{div}\{A(x) \hat{x}\}, \\
& F(t ; \rho, f, g)=\int_{S(t)}\left[f ( r ) \left\{2|\langle\hat{x}, A D v\rangle|^{2}-\langle\hat{x}, A \hat{x}\rangle(\langle D v, A \overline{D v}\rangle\right.\right. \\
& \left.\left.\left.\quad+\left(q_{1}+k_{1}\right)|v|^{2}\right)\right\}+g(x) \operatorname{Re}[\langle\hat{x}, A \overline{D v}\rangle v]\right] d S,
\end{aligned}
$$

where $\operatorname{Re}[w]$ means the real part of $w$.

Lemma 2.2. For $t>\mathbb{R}_{0}$ we have

$$
\begin{aligned}
& F(t ; \rho, f, g)=\exp (2 \rho(t)) \int_{S(t)}\left[f(r)\left\{2|\langle\hat{x}, A D u\rangle|^{2}-\langle\hat{x}, A \hat{x}\rangle\langle D u, A \overline{D u}\rangle\right\}\right. \\
&+\left\{2 \rho^{\prime} f\langle\hat{x}, A \hat{x}\rangle+g\right\} \operatorname{Re}[\langle\hat{x}, A \overline{D u}\rangle u]+\langle A \hat{x}, \hat{x}\rangle\left\{2 f \rho^{\prime 2}\langle\hat{x}, A \hat{x}\rangle\right. \\
&\left.\left.-f q_{1}+g \rho^{\prime}\right\}|u|^{2}\right] d S .
\end{aligned}
$$

Proof. We can easily obtain the above relation by direct calculation.

Lemma 2.3. For $t>s>R_{0}$ we have

$$
\begin{aligned}
F(t ; & \rho, f, g)-F(s ; \rho, f, g) \\
= & \int_{B(s, t)}\left[2\left(2 \rho^{\prime} f+f^{\prime}-r^{-1} f\right)|\langle A D v, \hat{x}\rangle|^{2}+\left(2 r^{-1} f+g\right.\right. \\
& \left.-f \operatorname{div}(A \hat{x})-\langle\hat{x}, A \hat{x}\rangle f^{\prime}\right)\langle A D v, \overline{D v}\rangle+2 r^{-1} f\left(|A D v|^{2}\right. \\
& -\langle A D v, \overline{D v}\rangle)+2 f \operatorname{Re}[\langle\hat{x},(\langle A D v, \nabla\rangle A) \overline{D v}\rangle] \\
& -f\langle(\langle\hat{x}, A \nabla\rangle A) D v, \overline{D v}\rangle+2 \operatorname{Re}\left[\left(f\left(q_{2}+k_{2}\right)+g \rho^{\prime}+2^{-1} \partial_{r} g\right)\langle\hat{x}, A \overline{D v}\rangle v\right] \\
& -2 f \operatorname{Im}[\langle B A \hat{x}, A \overline{D v}\rangle v]+\operatorname{Re}\left[\left\langle\left(\nabla-\hat{x} \partial_{r}\right) g, A \overline{D v}\right\rangle v\right] \\
& +\left\{\left(q_{1}+k_{1}\right)\left(g-f \operatorname{div}(A \hat{x})-f^{\prime}\langle\hat{x}, A \hat{x}\rangle\right)-f\left\langle\hat{x}, A \nabla\left(q_{1}+k_{1}\right)\right\rangle\right. \\
& \left.\left.+g\left(\operatorname{Re}\left[q_{2}\right]+k_{2}\right)\right\}|v|^{2}\right] d x,
\end{aligned}
$$

where $\operatorname{Im}[w]$ means the imaginary part of $w$.

Proof. See Lemma 2.1 of Uchiyama [2].

Lemma 2.4. Let $(0<) 2^{-1}(3-\alpha-\delta)<l<2^{-1}(3-\alpha)$. Then for any constant $\mu>\mu^{*}$ there exists some $R_{1}>R_{0}$ such that for any $t>S>R_{1}$ and any constant $m \geq 1$, we have

$$
\begin{aligned}
& F\left(t ; \mu r^{(3-\alpha) / 2}+m r^{l}, 1, \operatorname{div}(A \hat{x})-r^{-1} \gamma(x)\right) \\
& \quad \geq F\left(s ; \mu r^{(3-\alpha) / 2}+m r^{l}, 1, \operatorname{div}(A \hat{x})-r^{-1} \gamma(x)\right) .
\end{aligned}
$$


Proof. Let $\mu>\mu^{*}=\lim _{\eta_{\ddagger}} \mu^{(\eta)}$. By Lemma 2.1 there exists some $0<\eta_{0}<\delta$ such that $\mu>\mu^{\left(\eta_{0}\right)}$. Then there exists some $\varepsilon_{0}>0$ such that $\left(1+\varepsilon_{0}\right)^{-1} \mu \in M^{\left(\eta_{0}\right)}$. So

$$
\begin{aligned}
& \lim \sup _{r \rightarrow \infty}\left[r^{\alpha}\left\langle\hat{x}, A \nabla q_{1}\right\rangle+r r^{\alpha-1} q_{1}+(2 \mu(3-\alpha))^{-1}\left(1+\varepsilon_{0}\right) r^{(3 \alpha-1) / 2}\left|q_{2}\right|^{2}\right. \\
& \left.+\left(2-\eta_{0}-\gamma\right)^{-1} r^{\alpha-1}|B A x|^{2}-4^{-1} \mu^{2}(3-\alpha)^{2}(\gamma-\alpha+1)\right] \\
& <\limsup _{r \rightarrow \infty}\left[r^{\alpha}\left\langle\hat{x}, A \nabla q_{1}\right\rangle+r r^{\alpha-1} q_{1}+(2 \mu(3-\alpha))^{-1}\left(1+\varepsilon_{0}\right) r^{\left(3^{\alpha-1}\right) / 2}\left|q_{2}\right|^{2}\right. \\
& \left.+\left(2-\eta_{0}-\gamma\right)^{-1} r^{\alpha-1}|B A x|^{2}-4^{-1}\left(1+\varepsilon_{0}\right)^{-2} \mu^{2}(3-\alpha)^{2}(\gamma-\alpha+1)\right] \\
& <0 \text {. }
\end{aligned}
$$

Let

$$
\begin{aligned}
& \beta=(3-\alpha) / 2(>0), \rho(r)=\mu r^{\beta}+m r^{l}, f(r)=1, \\
& g(x)=\operatorname{div}(A(x) \hat{x})-r^{-1} \gamma(x), 0<\eta_{1}<\eta_{0}<\delta,
\end{aligned}
$$

where $m \geq 1$ and $\beta-2^{-1} \delta<l<\beta$ are constants. Let each $\varepsilon_{i}(r)(i=1,2, \cdots)$ be a positive function for $r>R_{0}$ which tends to 0 as $r \rightarrow \infty$. Noting

$$
\begin{aligned}
& |\langle A \hat{x}, \hat{x}\rangle-1|+r|\langle\hat{x}, A \nabla(\langle A \hat{x}, \hat{x}\rangle)\rangle| \leq \varepsilon_{1}(r), \\
& \operatorname{div}(A \hat{x})=O\left(r^{-1}\right) \quad \text { as } r \rightarrow \infty, \\
& g=O\left(r^{-1}\right) \quad \text { as } r \rightarrow \infty, \\
& \partial_{r} g=o\left(r^{\left(1-3 \alpha^{\alpha}\right) / 4}\right) \quad \text { as } r \rightarrow \infty, \\
& \left(\nabla-\hat{x} \partial_{r}\right) g=o\left(r^{-(1+\infty) / 2}\right) \quad \text { as } r \rightarrow \infty,
\end{aligned}
$$

we have, by direct calculation, the following estimates to the right hand side terms appeared in Lemma 2.3.

$$
\begin{aligned}
& 2\left(2 \rho^{\prime} f+f^{\prime}-r^{-1} f\right)|\langle A D v, \hat{x}\rangle|^{2} \\
& \quad \geq\left\{4 \mu \beta r^{\beta-1}+m l\left(4-\varepsilon_{2}(r)\right) r^{l-1}\right\}|\langle A D v, \hat{x}\rangle|^{2}, \\
& \left\{2 r^{-1} f+g-f \operatorname{div}(A \hat{x})-\langle\hat{x}, A \hat{x}\rangle f^{\prime}\right\}\langle A D v, \overline{D v}\rangle \\
& \quad=(2-r(x)) r^{-1}\langle A D v, \overline{D v}\rangle, \\
& 2 r^{-1} f\left(|A D v|^{2}-\langle A D v, \overline{D v}\rangle\right) \geq-\varepsilon_{3}(r) r^{-1}\langle A D v, \overline{D v}\rangle, \\
& 2 f \operatorname{Re}[\langle\hat{x},(\langle A D v, \nabla\rangle A) \overline{D v}\rangle] \geq-\varepsilon_{4}(r) r^{-1}\langle A D v, \overline{D v}\rangle, \\
& -f\langle(\langle\hat{x}, A \nabla\rangle A) D v, \overline{D v}\rangle \geq-\varepsilon_{5}(r) r^{-1}\langle A D v, \overline{D v}\rangle, \\
& 2 \operatorname{Re}\left[\left\{f\left(q_{2}+k_{2}\right)+g \rho^{\prime}+2^{-1} \partial_{r} g\right\}\langle\hat{x}, A \overline{D v}\rangle v\right] \\
& \quad \geq-\left(4 \mu \beta r^{\beta-1}+m l r^{l-1}\right)|\langle\hat{x}, A D v\rangle|^{2} \\
& \quad-r^{-\alpha}\left\{\left(1+2^{-1} \varepsilon_{0}\right)(4 \mu \beta)^{-1} r^{(3 \alpha-1) / 2}\left|q_{2}\right|^{2}+\varepsilon_{6}(r)\right\}|v|^{2} \\
& \quad-m l \varepsilon_{7}(r) r^{\beta+l-3}|v|^{2} \quad(, \text { where we use } \beta-2<(1-3 \alpha) / 4), \\
& -2 f \operatorname{Im}[\langle B A \hat{x}, A \overline{D v}\rangle v] \geq-\left(2-\eta_{1}-r(x)\right) r^{-1}\langle A D v, \overline{D v}\rangle
\end{aligned}
$$




$$
\begin{aligned}
& -\left(2-\eta_{1}-r(x)\right)^{-1} r^{-1}\left(1+\varepsilon_{\gamma}(r)\right)|B A x|^{2}|v|^{2}, \\
& \operatorname{Re}\left[\left\langle\left(\nabla-\hat{x} \partial_{r}\right) g, A \overline{D v}\right\rangle v\right] \geq-\varepsilon_{9}(r) r^{-1}\langle A D v, \overline{D v}\rangle-\varepsilon_{10}(r) r^{-\alpha}|v|^{2}, \\
& \left\{\left(q_{1}+k_{1}\right)\left(g-f \operatorname{div}(A \hat{x})-f^{\prime}\langle\hat{x}, A \hat{x}\rangle\right)-f\left\langle\hat{x}, A \nabla\left(q_{1}+k_{1}\right)\right\rangle\right\}|v|^{2} \\
& \geq r^{-\alpha}\left\{4^{-1} \mu^{2}(3-\alpha)^{2}(r(x)-\alpha+1)-r^{\alpha}\left\langle\hat{x}, A \nabla q_{1}\right\rangle-r r^{\alpha-1} q_{1}-\varepsilon_{11}(r)\right\}|v|^{2} \\
& \quad+\mu \beta m l\left(2 l+2 r-\alpha-1-\varepsilon_{12}(r)\right) r^{\beta+l-3}|v|^{2} \\
& \quad+(m l)^{2}\left(2 l+r-2-\varepsilon_{13}(r)\right) r^{2 l-3}|v|^{2}, \\
& g\left(\operatorname{Re}\left[q_{2}\right]+k_{2}\right)|v|^{2} \geq-r^{-\alpha}\left\{2^{-1} \varepsilon_{0}(4 \mu \beta)^{-1} r^{(3 \alpha-1) / 2}\left|q_{2}\right|^{2}+\varepsilon_{14}(r)\right\}|v|^{2} \\
& \quad-m l \varepsilon_{15}(r) r^{\beta+l-3}|v|^{2} .
\end{aligned}
$$

Then by Lemma 2.3 , we have

$$
\begin{aligned}
F\left(t ; \mu r^{\beta}+\right. & \left.m r^{l}, 1, \operatorname{div}(A \hat{x})-r r^{-1}\right)-F\left(s ; \mu r^{\beta}+m r^{l}, 1, \operatorname{div}(A \hat{x})-r r^{-1}\right) \\
\geq \int_{B(s, t)} & {\left[m l\left(3-\varepsilon_{16}(r)\right) r^{l-1}|\langle A D v, \hat{x}\rangle|^{2}\right.} \\
& +\left(\eta_{1}-\varepsilon_{16}(r)\right) r^{-1}\langle A D v, \overline{D v}\rangle+r^{-\alpha}\left\{4^{-1} \mu^{2}(3-\alpha)^{2}(r-\alpha+1)\right. \\
& \quad-\left(r^{\alpha}\left\langle\hat{x}, A D q_{1}\right\rangle+r r^{\alpha-1} q_{1}+\left(2-\eta_{0}-r\right)^{-1} r^{\alpha-1}|B A x|^{2}\right. \\
& \left.\left.+(4 \mu \beta)^{-1}\left(1+\varepsilon_{0}\right) r^{(3 \alpha-1) / 2}\left|q_{2}\right|^{2}+\varepsilon_{16}(r)\right)\right\}|v|^{2} \\
& +\left(2-\eta_{0}-\gamma\right)^{-1}\left(2-\eta_{1}-r\right)^{-1}\left\{\left(\eta_{0}-\eta_{1}\right)-\left(2-\eta_{0}-r\right) \varepsilon_{8}(r)\right\} r^{-1}|B A x|^{2}|v|^{2} \\
& +m l \mu \beta\left(2 l+2 \gamma-\alpha-1-\varepsilon_{16}(r)\right) r^{\beta+l-3}|v|^{2} \\
& \left.+(m l)^{2}\left(2 l+r-2-\varepsilon_{16}(r)\right) r^{2 l-3}|v|^{2}\right] d x .
\end{aligned}
$$

Noting

$$
\begin{aligned}
& 2 l+\gamma-2>2 l+\alpha-3+\delta>0, \\
& 2 l+2 \gamma-\alpha-1>(3-\alpha-\delta)+2(\alpha-1+\delta)-1-\alpha=\delta>0,
\end{aligned}
$$

there exists some $R_{1}>R_{0}$ such that for any $r \geq R_{1}$ and any $m \geq 1$ we have

$$
\begin{aligned}
& 3-\varepsilon_{16}(r) \geq 0, \\
& \eta_{1}-\varepsilon_{16}(r) \geq 0, \\
& 4^{-1} \mu^{2}(3-\alpha)^{2}(r-\alpha+1)-\left\{r^{\alpha}\left\langle\hat{x}, A \nabla q_{1}\right\rangle+r r^{\alpha-1} q_{1}+\left(2-\eta_{0}-r\right)^{-1} r^{\alpha-1}|B A x|^{2}\right. \\
& \left.\quad \quad+(4 \mu \beta)^{-1}\left(1+\varepsilon_{0}\right) r^{(3 \alpha-1) / 2}\left|q_{2}\right|^{2}+\varepsilon_{16}(r)\right\} \geq 0, \\
& \left(\eta_{0}-\eta_{1}\right)-\left(2-\eta_{0}-\gamma\right) \varepsilon_{8}(r) \geq 0, \\
& 2 l+2 \gamma-\alpha-1-\varepsilon_{16}(r) \geq 0, \\
& 2 l+r-2-\varepsilon_{16}(r) \geq 0 .
\end{aligned}
$$

Therefore we have the assertion.

Proof of Theorem 1.1 (1). We have only to show that for any $\mu>\mu^{*} \geq 0$ we have 


$$
\liminf _{R \rightarrow \infty} \exp \left(2 \mu R^{(3-\alpha) / 2}\right) \int_{S(R)}\left[|\langle A D u, \hat{x}\rangle|^{2}+\left\{1+\left(q_{1}\right)_{-}\right\}|u|^{2}\right] d S>0 .
$$

We shall prove the above inequality by contradiction. If we assume that this is not true, then there exists some $\mu_{0}>\mu^{*}$ such that

$$
\liminf _{R \rightarrow \infty} \exp \left(2 \mu_{0} R^{(3-\alpha) / 2}\right) \int_{S(R)}\left[|\langle A D u, \hat{x}\rangle|^{2}+\left\{1+\left(q_{1}\right)_{-}\right\}|u|^{2}\right] d S=0 .
$$

We choose constants $\mu$ and $l$ to satisfy $\mu_{0}>\mu>\mu^{*}$ and $0<(3-\alpha-\delta) / 2<l<(3-\alpha)$ 12. By Lemma 2.2 for any $m \geq 1$ there exists some constant $C_{3}>0$ such that for any $t \geq R_{0}$ we have

$$
\begin{aligned}
& F\left(t ; \mu r^{\beta}+m r^{l}, 1, \operatorname{div}(A \hat{x})-r r^{-1}\right) \\
& \quad \leq C_{3} \exp \left(2 \mu_{0} t^{\beta}\right) \int_{S(t)}\left[|\langle A D u, \hat{x}\rangle|^{2}+\left\{1+\left(q_{1}\right)_{-}\right\}|u|^{2}\right] d S,
\end{aligned}
$$

where $\beta=(3-\alpha) / 2>l$. Then we have

$$
\liminf _{t \rightarrow \infty} F\left(t ; \mu r^{\beta}+m r^{l}, 1, \operatorname{div}(A \hat{x})-r r^{-1}\right) \leq 0 .
$$

So letting $t \rightarrow \infty$ along suitable sequence in Lemma 2.4, we have for any $s \geq R_{1}$ and any $m \geq 1$

$$
F\left(s ; \mu r^{\beta}+m r^{l}, 1, \operatorname{div}(A \hat{x})-r r^{-1}\right) \leq 0 .
$$

On the other hand, since $\operatorname{supp}[u]$ is not a compact set in $\bar{\Omega}$, there exists some $R_{2}>R_{1}$ such that

By Lemma 2.2

$$
\int_{S\left(R_{2}\right)}|\langle\hat{x}, A \hat{x}\rangle|^{2}|u|^{2} d S>0
$$

$$
\exp \left(-2 \mu R_{2}^{(3-\alpha) / 2}-2 m R_{2}^{l}\right) F\left(R_{2} ; \mu r^{(3-\alpha) / 2}+m r^{l}, 1, \operatorname{div}(A \hat{x})-r r^{-1}\right)
$$

is a quadratic in $m$ of which coefficient of $m^{2}$ is

$$
2 l^{2} R_{2}^{2 l-2} \int_{S\left(R_{2}\right)}|\langle\hat{x}, A \hat{x}\rangle|^{2}|u|^{2} d S>0 .
$$

So there exists some $m_{0} \geq 1$ such that

$$
F\left(R_{2} ; \mu r^{(3-\alpha) / 2}+m_{0} r^{l}, 1, \operatorname{div}(A \hat{x})-r r^{-1}\right)>0,
$$

which leads to the contradiction.

\section{§3. Proof of Theorem 1.1 (2)}

In this section we assume all the conditions $(A) \sim(G)$. 
Lemma 3.1. Let a real-valued function $\zeta(t) \in C_{0}^{\infty}(-\infty, \infty)$ satisfy $\operatorname{supp}[\zeta]$ $\subset(0,1)$, and $\int_{0}^{1} \zeta^{2}(t) d t>0$, and let $\zeta_{R}(t)=\zeta(t-R)$ for $R>R_{0}$. Let $u(x)$ satisfy $\left.{ }^{*}\right)$, which is given in Theorem 1.1. Then there exist some constants $C_{4}>0$ and $R_{3}>R_{0}$ such that for any $R>R_{3}$ we have

$$
\begin{aligned}
& \int_{\Omega} \zeta_{R}^{2}(|x|)\left[|D u|^{2}+\left\{1+\left(q_{1}\right)_{-}\right\}|u|^{2}\right] d x \\
& \leq C_{4} \int_{B(R, R+1)} \exp \left(\nu^{*} r^{(3-\alpha) / 2}\right)|u|^{2} d x .
\end{aligned}
$$

Proof. We follow the similar arguments which have been given to prove Lemma 3.1 and Lemma 3.2 of Uchiyama [2]. Let $u_{\eta}(x)=\left\{|u(x)|^{2}+\eta^{2}\right\}^{1 / 2}$ for $\eta>0$. Then $u_{\eta} \in H_{\text {loc }}^{1}(\Omega)$ and $\left|\nabla u_{\eta}\right| \leq|D u|$, because of $u_{\eta} \nabla u_{\eta}=\operatorname{Re}[\bar{u} \nabla u]$ $=\operatorname{Re}[\bar{u} D u]$ and $|u| \leq\left|u_{\eta}\right|$. Noting $\zeta_{R} u_{\eta} \in H^{1}(\Omega)$ for $R>R_{0}$, for any $\varepsilon>0$ we have by $(\mathrm{G})$

$$
\begin{aligned}
\int_{\Omega} \zeta_{R}^{2}(|x|)\left(q_{1}+\operatorname{Re}\left[q_{2}\right]\right)_{-}\left|u_{\eta}\right|^{2} d x \\
\quad \leq a \int_{\Omega}\left|\nabla\left(\zeta_{R} u_{\eta}\right)\right|^{2} d x+C_{2} \int_{\Omega} \exp \left(\nu^{*} r^{(3-\alpha) / 2}\right)\left|\zeta_{R} u_{\eta}\right|^{2} d x \\
\quad \leq(1+\varepsilon) a \int_{\Omega} \zeta_{R}^{2}|D u|^{2} d x+C_{5} \int_{B(R, R+1)} \exp \left(\nu^{*} r^{(3-\alpha) / 2}\right)\left|u_{\eta}\right|^{2} d x,
\end{aligned}
$$

where $C_{5}=\max _{0 \leq t \leq 1}\left\{C_{2}|\zeta(t)|^{2}+\left(1+\varepsilon^{-1}\right) a\left|\zeta^{\prime}(t)\right|^{2}\right\}$. Letting $\eta \downarrow 0$ we have

$$
\begin{aligned}
& \int_{\Omega} \zeta_{R}^{2}\left(q_{1}+\operatorname{Re}\left[q_{2}\right]\right)_{-}|u|^{2} d x \\
& \quad \leq(1+\varepsilon) a \int_{\Omega} \zeta_{R}^{2}|D u|^{2} d x+C_{5} \int_{B(R, R+1)} \exp \left(\nu^{*} r^{(3-\alpha) / 2}\right)|u|^{2} d x .
\end{aligned}
$$

We choose $\varepsilon>0$ to satisfy $1>\varepsilon+(1+\varepsilon) a$. By (A1), (A2) and (A3) there exists some $R_{3}>R_{0}$ such that for any $r>R_{3}$ and any $\xi \in C^{n}$ we have $\langle A(x) \xi, \bar{\xi}\rangle \geq$ $(1-\varepsilon)|\xi|^{2}$. Since $u(x)$ satisfies $(*)$, we have for any $R>R_{3}$

$$
\begin{aligned}
0= & \operatorname{Re} \int_{\Omega} \zeta_{R}^{2}\left\{-\langle D, A D u\rangle+\left(q_{1}+q_{2}\right) u\right\} \bar{u} d x \\
= & \int_{\Omega} \zeta_{R}^{2}\left[\langle A D u, \overline{D u}\rangle+\left\{\left(q_{1}+\operatorname{Re}\left[q_{2}\right]\right)_{+}-\left(q_{1}+\operatorname{Re}\left[q_{2}\right]\right)_{-}\right\}|u|^{2}\right] d x \\
& \quad-\int_{\Omega}\left\{\zeta_{R} \zeta_{R}^{\prime} \operatorname{div}(A \hat{x})+\left(\zeta_{R} \zeta_{R}^{\prime}\right)^{\prime}\langle A \hat{x}, \hat{x}\rangle\right\}|u|^{2} d x \\
\geq & \{1-\varepsilon-(1+\varepsilon) a\} \int_{\Omega} \zeta_{R}^{2}|D u|^{2} d x-C_{6} \int_{B(R, R+1)} \exp \left(\nu^{*} r^{(3-\alpha) / 2}\right)|u|^{2} d x,
\end{aligned}
$$


where $C_{6}=C_{5}+C_{7} \max _{|\eta|>R_{3}}\{|\operatorname{div}(A \hat{x})|+\langle A \hat{x}, \hat{x}\rangle\} \quad$ and $\quad C_{7}=\max _{0 \leq t \leq 1}\left\{\left|\zeta(t) \zeta^{\prime}(t)\right|+\right.$ $\left.\left|\left(\zeta(t) \zeta^{\prime}(t)\right)^{\prime}\right|\right\}$. Then we have

$$
\int_{\Omega} \zeta_{R}^{2}|D u|^{2} d x \leq\{1-\varepsilon-(1+\varepsilon) a\}^{-1} C_{6} \int_{B(R, R+1,} \exp \left(\nu^{*} r^{\left(3-\alpha^{*}\right) / 2}\right)|u|^{2} d x .
$$

By the same arguments as given above, we have

$$
\int_{\Omega} \zeta_{R}^{2}\left(q_{1}\right)_{-}|u|^{2} d x \leq 2 b \int_{\Omega} \zeta_{R}^{2}|D u|^{2} d x+C_{8} \int_{B(R, R \mid 1)} \exp \left(\nu^{*} r^{(3-\alpha) / 2}\right)|u|^{2} d x
$$

where $C_{8}=\max _{0 \leq t \leq 1}\left\{C_{2}|\zeta(t)|^{2}+2 b\left|\zeta^{\prime}(t)\right|^{2}\right\}$. So we have the assertion.

Proof of Theorem 1.1 (2). Let $\mu>\mu^{*}+2^{-1} \nu^{*}$. We choose $\mu_{0}$ to satisfy $\mu-2^{-1} \nu^{*}>\mu_{0}>\mu^{*}$. By Lemma 3.1, we have for any $R>R_{3}$

$$
\begin{gathered}
\int_{\Omega} \zeta_{R}^{2}(|x|)\left[|D u|^{2}+\left\{1+\left(q_{1}\right)_{-}\right\}|u|^{2}\right] d x \\
\leq C_{4} \int_{B(R, R+1)} \exp \left(\nu^{*} r^{(3-\alpha) / 2}\right)|u|^{2} d x .
\end{gathered}
$$

By Theorem 1.1 (1), for any $L>0$ there exists some constant $R_{4}>R_{3}$ such that for any $t>R_{4}$

$$
L \exp \left(-2 \mu_{0} t^{(3-a) / 2}\right) \leq \int_{S(t)}\left[|\langle A D u, \hat{x}\rangle|^{2}+\left\{1+\left(q_{1}\right)_{-}\right\}|u|^{2}\right] d S .
$$

Then there exists some $C_{9}>0$ such that for any $R>R_{4}$ we have

$$
\begin{aligned}
& L\left\{\int_{0}^{1} \zeta^{2}(t) d t\right\} \exp \left(-2 \mu_{0}(R+1)^{(3-\alpha) / 2}\right) \\
& \quad \leq L \int_{R}^{R+1} \zeta_{R}^{2} \exp \left(-2 \mu_{0} t^{(3-\alpha) / 2}\right) d t \\
& \quad \leq \int_{\Omega} \zeta_{R}^{2}\left[|\langle A D u, \hat{x}\rangle|^{2}+\left\{1+\left(q_{1}\right)_{-}\right\}|u|^{2}\right] d S \\
& \quad \leq C_{9} \int_{B(R, R+1)} \exp \left(\nu^{*} r^{(3-\alpha) / 2}\right)|u|^{2} d x \\
& \quad \leq C_{9} \exp \left(\nu^{*}(R+1)^{(3-\alpha) / 2}\right) \int_{B(R, R+1)}|u|^{2} d x .
\end{aligned}
$$

Then for any $R>R_{4}$ we have

$$
\begin{aligned}
& \exp \left(2 \mu R^{(3-\alpha) / 2}\right) \int_{B(R, R-1)}|u|^{2} d x \\
& \geq C_{9}^{-1} L\left\{\int_{0}^{1} \zeta^{2}(t) d t\right\} \exp \left(\left\{2 \mu-\left(2 \mu_{0}+\nu^{*}\right)\left(1+R^{-1}\right)^{(3-\alpha) / 2}\right\} R^{(3-\alpha) / 2}\right),
\end{aligned}
$$


which leads the assertion.

\section{\$4. Proofs of Theorem 1.2 and Theorem 1.3}

Proof of Theorem 1.2. Under our weak condition (C3)', Lemma 2.3 is also true, if we replace $a_{i j}(x)$ with $\delta_{i j}$. (See Lemma 4.1 of Uchiyama [2].) Then Lemma 2.4 and the proof of Theorem 1.1 are also true by replacing $a_{i j}(x)$ with $\delta_{i j}$.

Proof of Theorem 1.3. Lemma 2.3 is also true with $a_{i j}(x)=\delta_{i j}$ and $b_{i}(x)$ $=0$. In the proof of Lemma 2.4 we have

$$
\begin{gathered}
\left\{2 r^{-1} f+g-f \operatorname{div}(A \hat{x})-f^{\prime}\langle\hat{x}, A \hat{x}\rangle\right\}\langle A D v, \overline{D v}\rangle=(2-r(x)) r^{-1}|\nabla v|^{2}, \\
2 r^{-1} f\left(|A D v|^{2}-\langle A D v, \overline{D v}\rangle\right)=0, \\
2 f \operatorname{Re}[\langle\hat{x},(\langle A D v, \nabla\rangle A) \overline{D v}\rangle]=0, \\
-f\langle(\langle\hat{x}, A \nabla\rangle A) D v, \overline{D v}\rangle=0, \\
-2 f \operatorname{Im}[\langle B A \hat{x}, A \overline{D v}\rangle v]=0, \\
\operatorname{Re}\left[\left\langle\left(\nabla-\hat{x} \partial_{r}\right) g, A \overline{D v}\right\rangle v\right] \geq-r^{-1} \sqrt{2-r(x)} p(x)|\nabla v||v| \\
\geq-(2-r) r^{-1}|\nabla v|^{2}-\varepsilon_{17}(r) r^{-\infty}|v|^{2} .
\end{gathered}
$$

Then Lemma 2.4 is also true in this case. Therefore the proof of Theorem 1.1 is also true.

\section{§5. Examples}

In the examples, except for Example 5.6, treated in this section, our $q_{1}(x)$ and $q_{2}(x)$ satisfy the followings:

(1) $q_{1}(x)$ is a real-valued function;

(2) $q_{2}(x)$ is a complex-valued function;

(3) there exists some $R_{0}>0$ such that for any compact set $K \subset\{x|| x \mid$ $\left.>R_{0}\right\}=\Omega, q_{1}(x), \partial_{r} q_{1}(x)$ and $q_{2}(x)$ are bounded in $K ;$

(4) there exists some constant $\alpha<3$ such that

$$
\begin{aligned}
& M=\left\{\mu>0 \mid \limsup _{r \rightarrow \infty}\left[r^{\alpha} \partial_{r} q_{1}+2 r^{\alpha-1} q_{1}\right.\right. \\
& \left.\left.\quad+(2 \mu(3-\alpha))^{-1} r^{(3 \alpha-1) / 2}\left|q_{2}\right|^{2}\right]<4^{-1} \mu^{2}(3-\alpha)^{3}\right\} \\
& \neq \phi ;
\end{aligned}
$$

(5) for any $\nu^{*}>0$ and any $\alpha<3$ there exists some constant $C_{10}>0$ such that for any $x \in \Omega$ we have 


$$
\left(q_{1}\right)_{-}(x)+\left(\operatorname{Re}\left[q_{2}\right]\right)_{-}(x) \leq C_{10} \exp \left(\nu^{*} r^{\left(3-\alpha^{*}\right) / 2}\right) .
$$

Then all the conditions in Theorem 1.3 are satisfied by $r(x)=2$. Now we can apply Theorem 1.3 to $u(x)$ satisfying

$$
(* * *)\left\{\begin{array}{l}
-\Delta u(x)+\left\{q_{1}(x)+q_{2}(x)\right\} u(x)=0 \text { in } \Omega, \\
u \in H_{\mathrm{loc}}^{2}(\Omega), \\
\operatorname{supp}[u] \text { is not a compact set in } \bar{\Omega} .
\end{array}\right.
$$

So we have by Theorem 1.3

$$
\lim _{R \rightarrow \infty} \exp \left(2 \mu R^{\beta}\right) \int_{R<|x|<R+1}|u|^{2} d x=\infty,
$$

where $\beta=(3-\alpha) / 2$, and $\mu>\mu^{*}=\inf M(\geq 0)$ is arbitrary, since the condition (G) holds for any $\nu^{*}>0$. In order to have a better estimate, we aim to choose $\beta>0$ as small as possible, namely to choose $\alpha$ as large as possible under the conditions that $M$ is not an empty set and $\alpha<3$. After the determination of $\alpha$ we calculate $\mu^{*}$.

Example 5.1. Let us consider

$$
\begin{aligned}
& q_{1}(x)=c r^{\theta}+V(x)-\lambda_{1}, \\
& q_{2}(x)=o\left(r^{\rho}\right)-i \lambda_{2},
\end{aligned}
$$

where $c>0, \theta \neq 0, \lambda_{1}, \lambda_{2}$ and $\rho$ are real constants, $i=\sqrt{-1}, q_{2}(x)$ is a complexvalued function and $V(x)$ is a real-valued function satisfying

$$
V(x)=o\left(r^{\theta}\right) \text { and } \partial_{r} V(x)=o\left(r^{\theta-1}\right) \quad \text { as } r \rightarrow \infty .
$$

Then we have

$$
\begin{array}{rl}
r^{\alpha} \partial_{r} q_{1} & +2 r^{\alpha-1} q_{1}+\{2 \mu(3-\alpha)\}^{-1} r^{(3 \alpha-1) / 2}\left|q_{2}\right|^{2} \\
=c & c(2+\theta) r^{\alpha+\theta-1}+o\left(r^{\alpha+\theta-1}\right)-2 \lambda_{1} r^{\alpha-1} \\
& +\{2 \mu(3-\alpha)\}^{-1} r^{(3 \alpha-1) / 2}\left|o\left(r^{\rho}\right)-i \lambda_{2}\right|^{2} .
\end{array}
$$

Noting that $\alpha+\theta-1 \geq 0$ for some $\alpha<3$ implies $2+\theta>0$, we have the followings.

Case I. $\lambda_{1}=\lambda_{2}=0$.

In this case we must examine the order relations between $3,1-\theta$ and $(1-4 \rho) / 3$.

(i) If $\max \{\theta, \rho\} \leq-2$, then $\beta>0$ is arbitrary and $\mu^{*}=0$, since $\min \{1-\theta$, $(1-4 \rho) / 3\} \geq 3$.

(ii) If $\theta>-2$ and $3 \theta \geq 4 \rho+2$, then $\beta=(2+\theta) / 2$ and $\mu^{*}=2 \sqrt{c}(2+\theta)^{-1}$, since $3>1-\theta$ and $(1-4 \rho) / 3 \geq 1-\theta$. 
(iii) If $\rho>-2$ and $4 \rho+2>3 \theta$, then $\beta=(4+2 \rho) / 3$ and $\mu^{*}=0$, since $\min \{3$, $1-\theta\}>(1-4 \rho) / 3$.

Case II. $\lambda_{1}>0$ and $\lambda_{2}=0$.

Note that $\alpha-1>(4 \rho+3 \alpha-1) / 2$ is equivalent to $\alpha<-1-4 \rho$. So if $\theta<0$, then we must examine the order relations between $3,-1-4 \rho$ and $(1-4 \rho) / 3$. And if $\theta>0$, we must examine the order relations between $3,1-\theta$ and $(1-4 \rho) / 3$.

(i) If $\theta<0$ and $\rho \leq-1$, then $\beta>0$ is arbitrary and $\mu^{*}=0$, since $-1-4 \rho$ $\geq 3$.

(ii) If $\theta<0$ and $-1<\rho \leq-1 / 2$, then $\beta=2+2 \rho(\leq 1)$ and $\mu^{*}=0$, since $3>-1-4 \rho \geq(1-4 \rho) / 3$.

(iii) If $\theta<0$ and $\rho>-1 / 2$, then $\beta=(4+2 \rho) / 3$ and $\mu^{*}=0$, since $3>(1-4 \rho)$ $13>-1-4 \rho$.

(iv) If $\theta>0$ and $3 \theta \geq 4 \rho+2$, then $\beta=(2+\theta) / 2$ and $\mu^{*}=2 \sqrt{c}(2+\theta)^{-1}$, since $3>1-\theta$ and $(1-4 \rho) / 3 \geq 1-\theta$.

(v) If $4 \rho+2>3 \theta>0$, then $\beta=(4+2 \rho) / 3$ and $\mu^{*}=0$, since $3>1-\theta>$ $(1-4 \rho) / 3$.

Case III. $\quad \lambda_{1}<0$ and $\lambda_{2}=0$.

In this case we must examine the order relations between $1,1-\theta$ and $(1-4 \rho) / 3$.

(i) If $\theta>0$ and $3 \theta \geq 4 \rho+2$, then $\beta=(2+\theta) / 2$ and $\mu^{*}=2 \sqrt{c}(2+\theta)^{-1}$, since $(1-4 \rho) / 3 \geq 1-\theta$ and $1>1-\theta$.

(ii) If $\rho>-1 / 2$ and $4 \rho+2>3 \theta$, then $\beta=(4+2 \rho) / 3$ and $\mu^{*}=0$, since $\min \{1,1-\theta\}>(1-4 \rho) / 3$.

(iii) If $\theta<0$ and $\rho \leq-1 / 2$, then $\beta=1$ and $\mu^{*}=\sqrt{-\lambda_{1}}$, since $1-\theta>1$ and $(1-4 \rho) / 3 \geq 1$.

Case IV. $\lambda_{2} \neq 0$ and $\lambda_{1} \in \mathbb{R}$ is arbitrary.

Noting that $\alpha-1>(3 \alpha-1) / 2$ is equivalent to $\alpha<-1(<1 / 3)$, we must examine the order relations between $1 / 3,1-\theta$ and $(1-4 \rho) / 3$.

(i) If $\theta>2 / 3$ and $3 \theta \geq 4 \rho+2$, then $\beta=(2+\theta) / 2$ and $\mu^{*}=2 \sqrt{c}(2+\theta)^{-1}$, since $1 / 3>1-\theta$ and $(1-4 \rho) / 3 \geq 1-\theta$.

(ii) If $\rho>0$ and $4 \rho+2>3 \theta$, then $\beta=(4+2 \rho) / 3$ and $\mu^{*}=0$, since $\min \{1 / 3$, $1-\theta\}>(1-4 \rho) / 3$.

(iii) If $\theta<2 / 3$ and $\rho \leq 0$, then $\beta=4 / 3$ and $\mu^{*}=2^{-11 / 3} 3^{4 / 3}\left|\lambda_{2}\right|^{2 / 3}$, since $(1-4 \rho) / 3 \geq 1 / 3$ and $1-\theta>1 / 3$.

(iv) If $\theta=2 / 3$ and $\rho \leq 0$, then $\beta=4 / 3$ and $\mu^{*}$ is the unique positive solution of the equation $\mu^{3}-2^{-4} 3^{2} c \mu-2^{-11} 3^{4}\left|\lambda_{2}\right|^{2}=0$, since $(1-4 \rho) / 3 \geq$ 
$1 / 3=1-\theta$

Remark 5.1. Let us consider the harmonic oscillator case

$$
\begin{aligned}
& q_{1}(x)=c r^{2}-\lambda_{1} \quad(c>0 \text { is a constant }) \\
& q_{2}(x)=0
\end{aligned}
$$

If

$$
\lambda_{1}=(2 l+n) \sqrt{c}
$$

for some integer $l \geq 0$, then (***) is satisfied by

$$
u_{l}(x)=\sum_{|k|=l} d_{k} H_{k_{1}}\left(c^{1 / 4} x_{1}\right) \cdots H_{k_{n}}\left(c^{1 / 4} x_{n}\right) \exp \left(-2^{-1} \sqrt{c} r^{2}\right),
$$

where $k=\left(k_{1}, \cdots, k_{n}\right),|k|=k_{1}+\cdots+k_{n}, k_{i}$ is a non-negative integer $(i=1, \cdots$, $n), d_{k}$ is a constant and $H_{j}(t)$ is the Hermite polynomial of degree $j$. Then for any $\varepsilon>0$ there exists some $C_{\varepsilon}>0$ and $R_{5}>0$ such that for any $|x|>R_{5}$ we have

$$
\left|u_{l}(x)\right|^{2} \leq C_{\varepsilon} \exp \left(-(1-\varepsilon) \sqrt{c} r^{2}\right) .
$$

On the other hand we have, from the results given in Case II, for any solution $u(x)$ of $(* * *)$ with $q_{1}(x)=c r^{2}-(2 l+n) \sqrt{c}$ and $q_{2}(x)=0$ and for any $\varepsilon>0$

$$
\lim _{R \rightarrow \infty} \exp \left((1+\varepsilon) \sqrt{c} R^{2}\right) \int_{R<|x|<R+1}|u|^{2} d x=\infty .
$$

If $l \geq 1$, then for any $R>0$ there exists some $x \in \mathbb{R}^{n}$ satisfying $|x|>R$ and $u_{l}(x)$ $=0$. So we cannot expect to have a pointwise estimate such as: for any solution $u(x)$ of $(* * *)$ with $q_{1}(x)=c r^{2}-\lambda_{1}\left(\lambda_{1}>0\right)$ and $q_{2}(x)=0$, and for any $\varepsilon>0$ we have

$$
\lim _{|x| \rightarrow \infty} \exp \left((1+\varepsilon) \sqrt{c}|x|^{2}\right)|u(x)|^{2}=\infty .
$$

Example 5.2. Let us consider

$$
\begin{aligned}
& q_{1}(x)=-c r^{\theta}+V(x)-\lambda_{1}, \\
& q_{2}(x)=o\left(r^{\rho}\right)-i \lambda_{2},
\end{aligned}
$$

where $c>0, \theta \neq 0, \rho, \lambda_{1}$ and $\lambda_{2}$ are real constants, $q_{2}(x)$ is a complex-valued function, and $V(x)$ is a real-valued function satisfying

$$
V(x)=o\left(r^{\theta}\right) \text { and } \partial_{r} V(x)=o\left(r^{\theta-1}\right) \text { as }|x| \rightarrow \infty .
$$

Then we have

$$
r^{\alpha} \partial_{r} q_{1}+2 r^{\alpha-1} q_{1}+\{2 \mu(3-\alpha)\}^{-1} r^{(3 \alpha-1) / 2}\left|q_{2}\right|^{2}
$$




$$
\begin{aligned}
=- & c(2+\theta) r^{\alpha+\theta-1}-2 \lambda_{1} r^{\alpha-1}+o\left(r^{\alpha+\theta-1}\right) \\
& +\{2 \mu(3-\alpha)\}^{-1} r^{(3 \alpha-1) / 2}\left|o\left(r^{\rho}\right)-i \lambda_{2}\right|^{2} .
\end{aligned}
$$

Noting that $\alpha+\theta-1 \geq 0$ for some $\alpha<3$ implies $2+\theta>0$, we have the following.

Case I. $\lambda_{1}=\lambda_{2}=0$.

Noting that $\alpha+\theta-1>(4 \rho+3 \alpha-1) / 2$ is equivalent to $\alpha<2 \theta-4 \rho-1$, we must examine the order relations between $3,2 \theta-4 \rho-1$ and $(1-4 \rho) / 3$.

(i) If $\theta \geq 2(1+\rho)$ or $\rho \leq-2$, then $\beta>0$ is arbitrary and $\mu^{*}=0$, since $\max \{2 \theta-4 \rho-1,(1-4 \rho) / 3\} \geq 3$.

(ii) If $2(1+\rho)>\theta>(2+4 \rho) / 3$, then $\beta=2+2 \rho-\theta$ and $\mu^{*}=0$, since $3>2 \theta-4 \rho-1>(1-4 \rho) / 3$.

(iii) If $\rho>-2$ and $2+4 \rho \geq 3 \theta$, then $\beta=(4+2 \rho) / 3$ and $\mu^{*}=0$, since $3>(1-4 \rho) / 3 \geq 2 \theta-4 \rho-1$.

Case II. $\lambda_{1}>0$ and $\lambda_{2}=0$.

If $\theta<0$, we must examine the order relations between $3,-1-4 \rho$ and $(1-4 \rho) / 3$. And if $\theta>0$, we must examine the order relations between 3 , $2 \theta-4 \rho-1$ and $(1-4 \rho) / 3$.

(i) If $\theta<0$ and $\rho \leq-1$, then $\beta>0$ is arbitrary and $\mu^{*}=0$, since $-1-4 \rho \geq 3$.

(ii) If $\theta<0$ and $-1<\rho \leq-1 / 2$, then $\beta=2+2 \rho$ and $\mu^{*}=0$, since $3>-1-4 \rho \geq(1-4 \rho) / 3$.

(iii) If $\theta<0$ and $\rho>-1 / 2$, then $\beta=(4+2 \rho) / 3$ and $\mu^{*}=0$, since $3>(1-4 \rho) / 3>-1-4 \rho$.

(iv) If $\theta>0$ and $\theta \geq 2(1+\rho)$, then $\beta>0$ is arbitrary and $\mu^{*}=0$, since $2 \theta-4 \rho-1 \geq 3$.

(v) If $\theta>0$ and $2(1+\rho)>\theta \geq(2+4 \rho) / 3$, then $\beta=2+2 \rho-\theta$ and $\mu^{*}=0$, since $3>2 \theta-4 \rho-1 \geq(1-4 \rho) / 3$.

(vi) If $2+4 \rho>3 \theta>0$, then $\beta=(4+2 \rho) / 3$ and $\mu^{*}=0$, since $3>(1-4 \rho) / 3>2 \theta-4 \rho-1$.

Case III. $\lambda_{1}<0$ and $\lambda_{2}=0$.

If $\theta<0$, we must examine the order relations between 1 and $(1-4 \rho) / 3$. If $\theta>0$, we must examine the order relations between $3,2 \theta-4 \rho-1$ and $(1-4 \rho)$ 13.

(i) If $\theta<0$ and $\rho \leq-1 / 2$, then $\beta=1$ and $\mu^{*}=\sqrt{-\lambda_{1}}$, since $(1-4 \rho) / 3 \geq 1$.

(ii) If $\theta<0$ and $\rho>-1 / 2$, then $\beta=(4+2 \rho) / 3$ and $\mu^{*}=0$, since $1>(1-4 \rho) / 3$. 
(iii) If $\theta>0$ and $\theta \geq 2(1+\rho)$, then $\beta>0$ is arbitrary and $\mu^{*}=0$, since $2 \theta-4 \rho-1 \geq 3$.

(iv) If $\theta>0$ and $2(1+\rho)>\theta \geq(2+4 \rho) / 3$, then $\beta=2+2 \rho-\theta$ and $\mu^{*}=0$, since $3>2 \theta-4 \rho-1 \geq(1-4 \rho) / 3$.

(v) If $2+4 \rho>3 \theta>0$, then $\beta=(4+2 \rho) / 3$ and $\mu^{*}=0$, since $3>(1-4 \rho) / 3$ $>2 \theta-4 \rho-1$.

Case IV. $\lambda_{2} \neq 0$ and $\lambda_{1} \in \mathbb{R}$ is arbitrary.

Note that $\alpha+\theta-1>\max \{(3 \alpha+4 \rho-1) / 2,(3 \alpha-1) / 2\}$ is equivalent to $\alpha<\min \{2 \theta-4 \rho-1,2 \theta-1\}$, and that $\alpha-1>(3 \alpha-1) / 2$ is equivalent to $\alpha<-1$ $(<1 / 3)$. So if $\rho \leq 0$, we must examine the order relations between $3,2 \theta-1$ and $1 / 3(\leq(1-4 \rho) / 3)$. And if $\rho>0$, we must examine the order relations between $3,2 \theta-4 \rho-1$ and $(1-4 \rho) / 3(\leq 1 / 3)$.

(i) If $\rho \leq 0$ and $\theta \geq 2$, then $\beta>0$ is arbitrary and $\mu^{*}=0$, since $2 \theta-1 \geq 3$.

(ii) If $\rho \leq 0$ and $2 / 3<\theta<2$, then $\beta=2-\theta$ and $\mu^{*}=4^{-1} c^{-1}\left(4-\theta^{2}\right)^{-1}\left|\lambda_{2}\right|^{2}$, since $3>2 \theta-1>1 / 3$.

(iii) If $\rho \leq 0$ and $\theta=2 / 3$, then $\beta=4 / 3$ and $\mu^{*}$ is the unique positive solution of the equation $\mu^{3}+2^{-4} 3^{2} c \mu-2^{-11} 3^{4}\left|\lambda_{2}\right|^{2}=0$, since $3>2 \theta-1=1 / 3$.

(iv) If $\rho \leq 0$ and $\theta<2 / 3$, then $\beta=4 / 3$ and $\mu^{*}=2^{-11 / 3} 3^{4 / 3}\left|\lambda_{2}\right|^{2 / 3}$, since $1 / 3>2 \theta-1$.

(v) If $\rho>0$ and $\theta \geq 2(1+\rho)$, then $\beta>0$ is arbitrary and $\mu^{*}=0$, since $2 \theta-4 \rho-1 \geq 3$.

(vi) If $\rho>0$ and $2(1+\rho)>\theta \geq(2+4 \rho) / 3$, then $\beta=2+2 \rho-\theta$ and $\mu^{*}=0$, since $3>2 \theta-4 \rho-1 \geq(1-4 \rho) / 3$.

(vii) If $\rho>0$ and $2+4 \rho>3 \theta$, then $\beta=(4+2 \rho) / 3$ and $\mu^{*}=0$, since $3>(1-4 \rho) / 3>2 \theta-4 \rho-1$.

Example 5.3. Let us consider

$$
\begin{aligned}
& q_{1}(x)=V(x)-\lambda_{1}, \\
& q_{2}(x)=o\left(r^{\rho}\right)-i \lambda_{2},
\end{aligned}
$$

where $\lambda_{1}, \lambda_{2}$ and $\rho$ are real constants, $q_{2}(x)$ is a complex-valued function, and $V(x)$ is a real-valued function satisfying

$$
V(x)=o(1) \text { and } \partial_{r} V(x)=o\left(r^{-1}\right) \text { as }|x| \rightarrow \infty .
$$

Then

$$
\begin{aligned}
& r^{\alpha} \partial_{r} q_{1}+2 r^{\alpha-1} q_{1}+\{2 \mu(3-\alpha)\}^{-1} r^{\left(3^{\alpha}-1\right) / 2}\left|q_{2}\right|^{2} \\
& \quad=-2 \lambda_{1} r^{\alpha-1}+o\left(r^{\alpha-1}\right)+\{2 \mu(3-\alpha)\}^{-1} r^{(3 \alpha-1) / 2}\left|o\left(r^{\rho}\right)-i \lambda_{2}\right|^{2} .
\end{aligned}
$$


Case I. $\quad \lambda_{1}=\lambda_{2}=0$.

We must examine the order relations between 1 and $(1-4 \rho) / 3$.

(i) If $\rho \leq-1 / 2$, then $\beta=1$ and $\mu^{*}=0$, since $(1-4 \rho) / 3 \geq 1$.

(ii) If $\rho>-1 / 2$, then $\beta=(4+2 \rho) / 3$ and $\mu^{*}=0$, since $1>(1-4 \rho) / 3$.

Case II. $\quad \lambda_{1}>0$ and $\lambda_{2}=0$.

We must examine the order relations between $3,-1-4 \rho$ and $(1-4 \rho) / 3$

(i) If $\rho \leq-1$, then $\beta>0$ is arbitrary and $\mu^{*}=0$, since $-1-4 \rho \geq 3$.

(ii) If $-1<\rho \leq-1 / 2$, then $\beta=2+2 \rho$ and $\mu^{*}=0$, since $3>-1-4 \rho \geq$ $(1-4 \rho) / 3$.

(iii) If $\rho>-1 / 2$, then $\beta=(4+2 \rho) / 3$ and $\mu^{*}=0$, since $3>(1-4 \rho) / 3>$ $-1-4 \rho$.

Case III. $\quad \lambda_{1}<0$ and $\lambda_{2}=0$.

We must examine the order relations between 1 and $(1-4 \rho) / 3$.

(i) If $\rho \leq-1 / 2$, then $\beta=1$ and $\mu^{*}=\sqrt{-\lambda_{1}}$, since $(1-4 \rho) / 3 \geq 1$.

(ii) If $\rho>-1 / 2$, then $\beta=(4+2 \rho) / 3$ and $\mu^{*}=0$, since $1>(1-4 \rho) / 3$.

Case IV. $\lambda_{2} \neq 0$ and $\lambda_{1} \in \mathbb{R}$ is arbitrary.

Noting that $\alpha-1>(3 \alpha-1) / 2$ is equivalent to $\alpha<-1 \quad(<1 / 3)$, we must examine the order relations between $1 / 3$ and $(1-4 \rho) / 3$.

(i) If $\rho \leq 0$, then $\beta=4 / 3$ and $\mu^{*}=2^{-11 / 3} 3^{4 / 3}\left|\lambda_{2}\right|^{2 / 3}$, since $(1-4 \rho) / 3 \geq 1 / 3$.

(ii) If $\rho>0$, then $\beta=(4+2 \rho) / 3$ and $\mu^{*}=0$, since $1 / 3>(1-4 \rho) / 3$.

Example 5.4. Let us consider

$$
\begin{aligned}
& q_{1}(x)=r^{\theta}\langle e, \hat{x}\rangle+V(x)-\lambda_{1}, \\
& q_{2}(x)=o\left(r^{\rho}\right)-i \lambda_{2},
\end{aligned}
$$

where $\theta, \rho, \lambda_{1}$ and $\lambda_{2}$ are real constants, $e \in \boldsymbol{R}^{n}(e \neq 0)$ is a constant vector, $q_{2}(x)$ is a complex-valued function, and $V(x)$ is a real-valued function satisfying

$$
V(x)=o\left(r^{\theta}\right) \text { and } \partial_{r} V(x)=o\left(r^{\theta-1}\right) \text { as }|x| \rightarrow \infty .
$$

Then

$$
\begin{aligned}
r^{\alpha} \partial_{r} q_{1} & +2 r^{\alpha-1} q_{1}+\{2 \mu(3-\alpha)\}^{-1} r^{(3 \alpha-1) / 2}\left|q_{2}\right|^{2} \\
= & (2+\theta)\left\langle e, \hat{x}>r^{\alpha+\theta-1}+o\left(r^{\alpha+\theta-1}\right)-2 \lambda_{1} r^{\alpha-1}\right. \\
& +\{2 \mu(3-\alpha)\}^{-1} r^{(3 \alpha-1) / 2}\left|o\left(r^{\rho}\right)-i \lambda_{2}\right|^{2} .
\end{aligned}
$$

Under the symbol of $\limsup _{r \rightarrow \infty},\langle e, \hat{x}\rangle$ behaves as like as $|e|$. So if $\theta \neq 0$, we have the same results as ones given in Example 5.1 by replacing $c$ with $|e|$. If $\theta=0$, we have the following. 
Case I. $\lambda_{1}=\lambda_{2}=0$ and $\theta=0$.

(i) If $\rho \leq-1 / 2$, then $\beta=1$ and $\mu^{*}=\sqrt{|e|}$, since $(1-4 \rho) / 3 \geq 1$.

(ii) If $\rho>-1 / 2$, then $\beta=(4+2 \rho) / 3$ and $\mu^{*}=0$, since $1>(1-4 \rho) / 3$.

Case II. $\lambda_{1}>0, \lambda_{2}=0$ and $\theta=0$.

(i) If $\rho \leq-1$ and $\lambda_{1}>|e|$, then $\beta>0$ is arbitrary and $\mu^{*}=0$, since $-1-4 \rho \geq 3$.

(ii) If $-1<\rho \leq-1 / 2$ and $\lambda_{1}>|e|$, then $\beta=2+2 \rho$ and $\mu^{*}=0$, since $3>-1-4 \rho \geq(1-4 \rho) / 3$.

(iii) If $\rho>-1 / 2$ and $\lambda_{1}>|e|$, then $\beta=(4+2 \rho) / 3$ and $\mu^{*}=0$, since $3>(1-4 \rho) / 3>-1-4 \rho$.

(iv) If $\rho \leq-2$ and $\lambda_{1}=|e|$, then $\beta>0$ is arbitrary and $\mu^{*}=0$, since $(1-4 \rho) / 3 \geq 3$.

(v) If $\rho>-2$ and $\lambda_{1}=|e|$, then $\beta=(4+2 \rho) / 3$ and $\mu^{*}=0$, since $3>(1-4 \rho) / 3$.

(vi) If $\rho \leq-1 / 2$ and $0<\lambda_{1}<|e|$, then $\beta=1$ and $\mu^{*}=\sqrt{\mid e_{\mid}-\lambda_{1}}$, since $(1-4 \rho) / 3 \geq 1$.

(vii) If $\rho>-1 / 2$ and $0<\lambda_{1}<|e|$, then $\beta=(4+2 \rho) / 3$ and $\mu^{*}=0$, since $1>(1-4 \rho) / 3$.

Case III. $\lambda_{1}<0, \lambda_{2}=0$ and $\theta=0$.

(i) If $\rho \leq-1 / 2$, then $\beta=1$ and $\mu^{*}=\sqrt{|e|-\lambda_{1}}$, since $(1-4 \rho) / 3 \geq 1$.

(ii) If $\rho>-1 / 2$, then $\beta=(4+2 \rho) / 3$ and $\mu^{*}=0$, since $1>(1-4 \rho) / 3$.

Case $I V . \quad \lambda_{2} \neq 0, \lambda_{1} \in \mathbb{R}$ is arbitrary and $\theta=0$.

(i) If $\rho \leq 0$, then $\beta=4 / 3$ and $\mu^{*}=2^{-11 / 3} 3^{4 / 3}\left|\lambda_{2}\right|^{2 / 3}$, since $(1-4 \rho) / 3 \geq 1 / 3$.

(ii) If $\rho>0$, then $\beta=(4+2 \rho) / 3$ and $\mu^{*}=0$, since $1 / 3>(1-4 \rho) / 3$.

Example 5.5. Let us consider

$$
q_{1}(x)+q_{2}(x)=a r^{\sigma} \sin b r^{\tau}+V(x)-\left(\lambda_{1}+i \lambda_{2}\right),
$$

where $a, b, \sigma, \tau, \lambda_{1}$ and $\lambda_{2}$ are real constants satisfying $a b \neq 0$ and $\tau>0$, and $V(x)$ is a real-valued function satisfying

$$
V(x)=o\left(r^{\sigma}\right) \text { and } \partial_{r} V(x)=o\left(r^{\sigma+\tau-1}\right) \text { as }|x| \rightarrow \infty .
$$

For this example we should consider the following both cases:

and

$$
\begin{aligned}
& q_{1}(x)=a r^{\sigma} \sin b r^{\tau}+V(x)-\lambda_{1}, \\
& q_{2}(x)=-i \lambda_{2},
\end{aligned}
$$

$$
q_{1}(x)=-\lambda_{1}
$$




$$
q_{2}(x)=a r^{\sigma} \sin b r^{\tau}+V(x)-i \lambda_{2} .
$$

In the first case we have

$$
\begin{gathered}
r^{\alpha} \partial_{r} q_{1}+2 r^{\alpha-1} q_{1}+\{2 \mu(3-\alpha)\}^{-1} r^{(3 \alpha-1) / 2}\left|q_{2}\right|^{2} \\
=a b \tau r^{\alpha+\sigma+\tau-1} \cos b r^{\tau}+o\left(r^{\alpha+\sigma+\tau-1}\right)-2 \lambda_{1} r^{\alpha-1} \\
+\{2 \mu(3-\alpha)\}^{-1}\left|\lambda_{2}\right|^{2} r^{(3 \alpha-1) / 2} .
\end{gathered}
$$

In the second case we have

$$
\begin{aligned}
& r^{\alpha} \partial_{r} q_{1}+2 r^{\alpha-1} q_{1}+\{2 \mu(3-\alpha)\}^{-1} r^{(3 \alpha-1) / 2}\left|q_{2}\right|^{2} \\
& \quad=-2 \lambda_{1} r^{\alpha-1}+\{2 \mu(3-\alpha)\}^{-1}\left|a r^{\sigma} \sin b r^{\tau}+o\left(r^{\sigma}\right)-i \lambda_{2}\right|^{2} .
\end{aligned}
$$

Comparing two results which are obtained from these both cases, we choose the better values of $\beta>0$ and $\mu^{*} \geq 0$. Then we have the following.

Case I. $\lambda_{1}=\lambda_{2}=0$.

(i) If $\sigma \leq-2$, then $\beta>0$ is arbitrary and $\mu^{*}=0$.

(ii) If $-2<\sigma<3 \tau-2$, then $\beta=(4+2 \sigma) / 3$ and $\mu^{*}=2^{-7 / 3} 3^{4 / 3}|a|^{2 / 3}(2+\sigma)^{-4 / 3}$.

(iii) If $\sigma=3 \tau-2$ and $0<\tau<2^{-1}|a|^{1 / 2}|b|^{-3 / 2}$, then $\beta=2 \tau$ and $\mu^{*}=$ $4^{-1}|a b|^{1 / 2} \tau^{-1}$.

(iv) If $\sigma=3 \tau-2$ and $\tau \geq 2^{-1}|a|^{1 / 2}|b|^{-3 / 2}$, then $\beta=2 \tau$ and $\mu^{*}=$ $2^{-7 / 3} \tau^{-4 / 3}|a|^{2 / 3}$.

(v) If $\sigma>3 \tau-2$, then $\beta=(2+\sigma+\tau) / 2$ and $\mu^{*}=2|a b \tau|^{1 / 2}(2+\sigma+\tau)^{-3 / 2}$.

Case II. $\lambda_{1}>0$ and $\lambda_{2}=0$.

(i) If $\sigma+\tau>0$ and $\sigma>3 \tau-2$, then $\beta=(2+\sigma+\tau) / 2$ and $\mu^{*}=$ $2|a b \tau|^{1 / 2}(2+\sigma+\tau)^{-3 / 2}$.

(ii) If $-1 / 2<\sigma<3 \tau-2$, then $\beta=(4+2 \sigma) / 3$ and $\mu^{*}=2^{-7 / 3} 3^{4 / 3}|a|^{2 / 3}(2+$ $\sigma)^{-4 / 3}$.

(iii) If $\sigma+\tau>0$ and $-1<\sigma<-1 / 2$, then $\beta=2+2 \sigma$ and $\mu^{*}=0$.

(iv) If $\sigma+\tau<0$ or $\sigma \leq-1$, then $\beta>0$ is arbitrary and $\mu^{*}=0$.

(v) If $\sigma=-1 / 2$ and $\tau>1 / 2$, then $\beta=1$ and $\mu^{*}$ is the unique positive solution of the equation $\mu^{3}+\lambda_{1} \mu-8^{-1} a^{2}=0$.

(vi) If $\sigma=3 \tau-2$ and $\tau>1 / 2$, then $\beta=2 \tau$ and $\mu^{*}$ is some positive constant, which is classified into several cases.

(vii) If $\sigma+\tau=0$ and $-1<\sigma<0$, then the determinations of $\beta$ and $\mu^{*}$ are classified into several cases.

Case III. $\lambda_{1}<0$ and $\lambda_{2}=0$.

(i) If $\sigma+\tau>0$ and $\sigma>3 \tau-2$, then $\beta=(2+\sigma+\tau) / 2$ and $\mu^{*}=$ $2|a b \tau|^{1 / 2}(2+\sigma+\tau)^{-3 / 2}$. 
(ii) If $-1 / 2<\sigma<3 \tau-2$, then $\beta=(4+2 \sigma) / 3$ and $\mu^{*}=2^{-7 / 3} 3^{4 / 3}|a|^{2 / 3}(2+$ $\sigma)^{-4 / 3}$

(iii) If $\sigma+\tau<0$ or $\sigma<-1 / 2$, then $\beta=1$ and $\mu^{*}=\sqrt{-\lambda_{1}}$.

(iv) If $\sigma=-1 / 2$ and $\tau>1 / 2$, then $\beta=1$ and $\mu^{*}$ is the unique positive solution of the equation $\mu^{3}+\lambda_{1} \mu-8^{-1} a^{2}=0$.

(v) If $\sigma+\tau=0$ and $-1 / 2<\sigma<0$, then $\beta=1$ and $\mu^{*}=2^{-1 / 2} \sqrt{|a b \tau|-2 \lambda_{1}}$.

(vi) If $\sigma=3 \tau-2$ and $\tau \geq 1 / 2$, then $\beta=2 \tau$ and $\mu^{*}$ is some positive constant, which is classified into several cases.

Case IV. $\lambda_{2} \neq 0$ and $\lambda_{1} \in \mathbb{R}$ is arbitrary.

(i) If $\sigma+\tau>2 / 3$ and $\sigma>3 \tau-2$, then $\beta=(2+\sigma+\tau) / 2$ and $\mu^{*}=$ $2|a b \tau|^{1 / 2}(2+\sigma+\tau)^{-3 / 2}$.

(ii) If $0<\sigma<3 \tau-2$, then $\beta=(4+2 \sigma) / 3$ and $\mu^{*}=2^{-7 / 3} 3^{4 / 3}|a|^{2 / 3}(2+\sigma)^{-4 / 3}$.

(iii) If $\sigma+\tau<2 / 3$ or $\sigma<0$, then $\beta=4 / 3$ and $\mu^{*}=2^{-11 / 3} 3^{4 / 3}\left|\lambda_{2}\right|^{2 / 3}$.

(iv) If $\sigma=0$ and $\tau>2 / 3$, then $\beta=4 / 3$ and $\mu^{*}=2^{-11 / 3} 3^{4 / 3}\left\{|a|^{2}+\left|\lambda_{2}\right|^{2}\right\}^{1 / 3}$.

(v) If $\sigma+\tau=2 / 3$ and $0<\sigma<2 / 3$, then $\beta=4 / 3$ and $\mu^{*}$ is the unique positive solution of the equation $\mu^{3}-2^{-7} 3^{3}|a b \tau| \mu-2^{-11} 3^{4}\left|\lambda_{2}\right|^{2}=0$.

(vi) If $\sigma=3 \tau-2$ and $\tau \geq 2 / 3$, then $\beta=2 \tau$ and $\mu^{*}$ is some positive constant, which is classified into several cases.

Remark 5.2. Let $\tau<0$ in Example 5.5. Then we have

$$
\begin{aligned}
& a r^{\sigma} \sin b r^{\tau}=a b r^{\sigma+\tau}+V_{1}(x), \\
& V_{1}(x)=a b r^{\sigma+\tau}\left\{\left(b r^{\tau}\right)^{-1} \sin b r^{\tau}-1\right\},
\end{aligned}
$$

where $V_{1}(x)$ satisfies

$$
V_{\mathrm{J}}(x)=o\left(r^{\sigma+\tau}\right) \text { and } \partial_{r} V_{1}(x)=o\left(r^{\sigma+\tau-1}\right) \text { as }|x| \rightarrow \infty .
$$

So if $V(x)$ in Example 5.5 satisfies a more strict condition than former one

$$
V(x)=o\left(r^{\sigma+\tau}\right) \text { and } \partial_{r} V(x)=o\left(r^{\sigma+\tau-1}\right) \text { as }|x| \rightarrow \infty,
$$

the problem is reduced to Example 5.1 or Example 5.2 by replacing $\sigma+\tau$ with $\theta$.

Example 5.6. Let us consider

$$
\begin{aligned}
& q_{1}(x)=V_{1}(x)+V_{2}(x)-\lambda_{1}, \\
& q_{2}(x)=o\left(r^{\rho}\right)-i \lambda_{2},
\end{aligned}
$$

where $\rho, \lambda_{1}$ and $\lambda_{2}$ are real constants, $q_{2}(x)$ is a complex-valued function, and $V_{2}(x)$ is a real-valued function satisfying 


$$
V_{2}(x)=o\left(r^{\theta}\right) \text { and } \partial_{r} V_{2}(x)=o\left(r^{\theta-1}\right) \text { as }|x| \rightarrow \infty
$$

for some real constant $\theta$. For a real-valued function $V_{1}(x)$ we assume:

(6) for any $w \in H_{\text {loc }}^{1}(\Omega)$ (, where $\Omega=\left\{x|| x \mid>R_{0}\right\}$,) we have $V_{1}|w|^{2} \in$ $L_{\text {loc }}^{1}(\Omega)$ and $\left(\partial_{r} V_{1}\right)|w|^{2} \in L_{\text {loc }}^{1}(\Omega)$

(7) there exists some constant $\gamma_{0} \leq 2$ such that for any $\alpha<r_{0}+1$

$$
\limsup _{r \rightarrow \infty}\left[r^{\alpha} \partial_{r} V_{1}(x)+r_{0} r^{\alpha-1} V_{1}(x)\right] \leq 0 ;
$$

(8) for any $\nu^{*}>0$ and any $\alpha<\gamma_{0}+1$ there exist some constants $0<b<1$ and $C_{2} \geq 0$ such that for any $w \in H^{1}(\Omega)$ having a compact support in $\Omega$

$$
\int_{\Omega}\left(V_{1}\right)_{-}|w|^{2} d x \leq b \int_{\Omega}|\nabla w|^{2} d x+C_{2} \int_{\Omega} \exp \left(\nu^{*} r^{(3-\infty) / 2}\right)|w|^{2} d x .
$$

Noting that we have $(f+g)_{-}(x) \leq(f)_{-}(x)+(g)_{-}(x)$ for any real-valued functions $f(x)$ and $g(x), q_{1}(x)$ and $q_{2}(x)$ given above satisfy (G). Let for $\alpha<\gamma_{0}+1$

$$
\begin{aligned}
M= & \left\{\mu>0 \mid \limsup _{r \rightarrow \infty}\left[r^{\alpha} \partial_{r} q_{1}+r_{0} r^{\alpha-1} q_{1}+(2 \mu(3-\alpha))^{-1} r^{(3 \alpha-1) / 2}\left|q_{2}\right|^{2}\right]\right. \\
& \left.<4^{-1} \mu^{2}(3-\alpha)^{2}\left(\gamma_{0}+1-\alpha\right)\right\}, \\
\tilde{M}= & \left\{\mu>0 \mid \lim _{r \rightarrow \infty} \sup \left[o\left(r^{\alpha+\theta-1}\right)-\gamma_{0} \lambda_{1} r^{\alpha-1}\right.\right. \\
& \left.\quad+(2 \mu(3-\alpha))^{-1} r^{(3 \alpha-1) / 2}\left|o\left(r^{\rho}\right)-i \lambda_{2}\right|^{2}\right] \\
& \left.<4^{-1} \mu^{2}(3-\alpha)^{2}\left(\gamma_{0}+1-\alpha\right)\right\}, \\
\mu^{*}= & \inf M \text { and } \mu^{* *}=\inf \tilde{M} .
\end{aligned}
$$

Then $(\mathrm{F})^{\prime}$ in Theorem 1.3 is satisfied by the above $q_{1}(x), q_{2}(x)$ and $r(x)=r_{0}$. By Theorem 1.3 we have for any $u(x)$ satisfying $\left({ }^{* *}\right)$

$$
\lim _{R \rightarrow \infty} \exp \left(2 \mu R^{\beta}\right) \int_{R<|x|<R+1}|u|^{2} d x=\infty,
$$

where $\beta=(3-\alpha) / 2>\left(2-\gamma_{0}\right) / 2 \geq 0$ by $\alpha<\gamma_{0}+1 \leq 3$, and $\mu>\mu^{*}$ is arbitrary. Now we choose $\beta$ as small as possible under the conditions that $\tilde{M}$ is not an empty set and $\alpha<\gamma_{0}+1(\leq 3)$. After the determination of $\beta$ we calculate $\mu^{* *}\left(\geq \mu^{*}\right.$ $\geq 0$ ).

Case I. $\quad \gamma_{0} \lambda_{1}=\lambda_{2}=0$.

We must examine the order relations between $\gamma_{0}+1,1-\theta$ and $(1-4 \rho) / 3$.

(i) If $\theta \leq-\gamma_{0}$ and $4 \rho \leq-\left(3 \gamma_{0}+2\right)$, then $\beta>\left(2-\gamma_{0}\right) / 2$ is arbitrary and $\mu^{* *}=0$, since $\min \{1-\theta,(1-4 \rho) / 3\} \geq \gamma_{0}+1$.

(ii) If $\theta>-\gamma_{0}$ and $3 \theta \geq 4 \rho+2$, then $\beta=(2+\theta) / 2$ and $\mu^{* * *}=0$, since $\gamma_{0}+$ $1>1-\theta$ and $(1-4 \rho) / 3 \geq 1-\theta$.

(iii) If $4 \rho>-\left(3 \gamma_{0}+2\right)$ and $3 \theta<4 \rho+2$, then $\beta=(4+2 \rho) / 3$ and $\mu^{* *}=0$, 
since $\min \left\{\gamma_{0}+1,1-\theta\right\}>(1-4 \rho) / 3$.

Case II. $\gamma_{0}<0, \lambda_{1} \neq 0$ and $\lambda_{2}=0$.

Since $\lim _{1 \rightarrow \infty} r^{\alpha-1}=0$ for any $\alpha<\gamma_{0}+1(<1)$, we have the same results as Case $\mathbb{I}$.

Case III. $\gamma_{0}>0, \lambda_{1}>0$ and $\lambda_{2}=0$.

If $\theta>0$, we must examine the order relations between $\left(r_{0}+1>1>\right) 1-\theta$ and $(1-4 \rho) / 3$. And if $\theta \leq 0$, we must examine the order relations between $r_{0}+1(>1),-1-4 \rho$ and $(1-4 \rho) / 3$.

(i) If $\theta>0$ and $3 \theta \geq 4 \rho+2$, then $\beta=(2+\theta) / 2$ and $\mu^{* * *}=0$, since $(1-4 \rho) / 3$ $\geq 1-\theta$.

(ii) If $4 \rho+2>3 \theta>0$, then $\beta=(4+2 \rho) / 3$ and $\mu^{* *}=0$, since $1-\theta>$ $(1-4 \rho) / 3$.

(iii) If $\theta \leq 0$ and $4 \rho \leq-\left(\gamma_{0}+2\right)$, then $\beta>\left(2-\gamma_{0}\right) / 2$ is arbitrary and $\mu^{* *}=0$, since $-1-4 \rho \geq r_{0}+1$.

(iv) If $\theta \leq 0$ and $-\left(\gamma_{0}+2\right)<4 \rho \leq-2$, then $\beta=2 \rho+2$ and $\mu^{* *}=0$, since $r_{0}+1>-1-4 \rho \geq(1-4 \rho) / 3$.

(v) If $\theta \leq 0$ and $\rho>-1 / 2$, then $\beta=(4+2 \rho) / 3$ and $\mu^{* * *}=0$, since $\gamma_{0}+1$ $>(1-4 \rho) / 3>-1-4 \rho$.

Case $I V . \quad r_{0}>0, \lambda_{1}<0$ and $\lambda_{2}=0$.

We must examine the order relations between $1\left(<\gamma_{0}+1\right), 1-\theta$ and $(1-4 \rho) / 3$.

(i) If $\theta>0$ and $3 \theta \geq 4 \rho+2$, then $\beta=(2+\theta) / 2$ and $\mu^{* *}=0$, since $(1-4 \rho) / 3$ $\geq 1-\theta$ and $1>1-\theta$.

(ii) If $\rho>-1 / 2$ and $4 \rho+2>3 \theta$, then $\beta=(4+2 \rho) / 3$ and $\mu^{* *}=0$, since $\min \{1,1-\theta\}>(1-4 \rho) / 3$.

(iii) If $\theta \leq 0$ and $\rho \leq-1 / 2$, then $\beta=1$ and $\mu^{* *}=\sqrt{-\lambda_{1}}$, since $\min \{(1-4 \rho) / 3,1-\theta\} \geq 1$.

Case $V . \quad-2 / 3<r_{0} \leq 2, \lambda_{2} \neq 0$ and $\lambda_{1} \in \mathbb{R}$ is arbitrary.

Noting that $\alpha-1>(3 \alpha-1) / 2$ is equivalent to $\alpha<-1\left(<1 / 3<\gamma_{0}+1\right)$, we must examine the order relations between $1 / 3,1-\theta$ and $(1-4 \rho) / 3$.

(i) If $\theta>2 / 3$ and $3 \theta \geq 4 \rho+2$, then $\beta=(2+\theta) / 2$ and $\mu^{* *}=0$, since $1 / 3$ $>1-\theta$ and $(1-4 \rho) / 3 \geq 1-\theta$.

(ii) If $\rho>0$ and $4 \rho+2>3 \theta$, then $\beta=(4+2 \rho) / 3$ and $\mu^{* *}=0$, since $\min \{1 / 3$, $1-\theta\}>(1-4 \rho) / 3$.

(iii) If $\theta \leq 2 / 3$ and $\rho \leq 0$, then $\beta=4 / 3$ and $\mu^{* * *}=2^{-8 / 3} 3^{4 / 3}\left(3 \gamma_{0}+2\right)^{-1 / 3}\left|\lambda_{2}\right|^{2 / 3}$, since $\min \{(1-4 \rho) / 3,1-\theta\} \geq 1 / 3$.

Case VI. $r_{0} \leq-2 / 3, \lambda_{2} \neq 0$ and $\lambda_{1} \in \mathbb{R}$ is arbitrary. 
Since $\lim _{r \rightarrow \infty} r^{\alpha-1}=\lim _{r \rightarrow \infty} r^{(3 \alpha-1) / 2}=0$ for any $\alpha<r_{0}+1 \leq 1 / 3$, we have the same results as Case I.

Remark 5.3. If $V_{1}(x)$ is a homogeneous function of degree $-\gamma_{0}$, then we have $\partial_{r} V_{1}(x)+\gamma_{0} r^{-1} V_{1}(x)=0$. Let

$$
V_{1}(x)=\sum_{1 \leq i \leq N} Z_{i}\left|x^{(i)}\right|^{-1}+\sum_{1 \leq i<j \leq N} Z_{i j}\left|x^{(i)}-x^{(j)}\right|^{-1},
$$

where $Z_{i}$ and $Z_{i j}$ are real constants, $x^{(i)} \in \boldsymbol{R}^{3}$, and $x=\left(x^{(1)}, \cdots, x^{(N)}\right) \in \mathbb{R}^{3 N}$. This $V_{1}(x)$ is a homogeneous function of degree -1 . Then $V_{1}(x)$ satisfies (6), (7) and (8) with $\gamma_{0}=1$.

\section{References}

[1] Bardos C. and Merigot M., Asymptotic decay of the solution of a second-order elliptic equation in an unbounded domain. Applications to the spectral properties of a Hamiltonian, Proc. Roy. Soc. Edinburgh 76A (1977), 323-344.

[2] Uchiyama J., Lower bounds of decay order of eigenfunctions of second-order elliptic operators, Publ. RIMS. Kyoto Univ. 21 (1985), 1281-1297. 